\title{
Maps of Dust Infrared Emission for Use in Estimation of Reddening and Cosmic Microwave Background Radiation Foregrounds
}

\section{Citation}

Schlegel, David J., Douglas P. Finkbeiner, and Marc Davis. 1998. "Maps of Dust Infrared Emission for Use in Estimation of Reddening and Cosmic Microwave Background Radiation Foregrounds." The Astrophysical Journal 500 (2) (June 20): 525-553. doi:10.1086/305772.

\section{Published Version}

$10.1086 / 305772$

\section{Permanent link}

http://nrs.harvard.edu/urn-3:HUL.InstRepos:33461989

\section{Terms of Use}

This article was downloaded from Harvard University's DASH repository, and is made available under the terms and conditions applicable to Other Posted Material, as set forth at http:// nrs.harvard.edu/urn-3:HUL.InstRepos:dash.current.terms-of-use\#LAA

\section{Share Your Story}

The Harvard community has made this article openly available.

Please share how this access benefits you. Submit a story.

\section{Accessibility}


ThE ASTROPHYSICAL JOURNAL, 500:525-553, 1998 June 20

(C) 1998. The American Astronomical Society. All rights reserved. Printed in U.S.A.

\title{
MAPS OF DUST INFRARED EMISSION FOR USE IN ESTIMATION OF REDDENING AND COSMIC MICROWAVE BACKGROUND RADIATION FOREGROUNDS
}

\author{
DAVID J. SCHLEGEL \\ University of Durham, Department of Physics, South Road, Durham DH1 3LE, UK; D.J.Schlegel@durham.ac.uk \\ AND \\ Douglas P. Finkbeiner AND Marc DAVIS \\ University of California at Berkeley, Departments of Physics and Astronomy, 601 Campbell Hall, Berkeley, CA 94720; \\ dfink@astro.berkeley.edu,marc@coma.berkeley.edu \\ Received 1997 October 17; accepted 1998 January 28
}

\begin{abstract}
We present a full-sky $100 \mu \mathrm{m}$ map that is a reprocessed composite of the COBE/DIRBE and IRAS/ ISSA maps, with the zodiacal foreground and confirmed point sources removed. Before using the ISSA maps, we remove the remaining artifacts from the IRAS scan pattern. Using the DIRBE 100 and $240 \mu \mathrm{m}$ data, we have constructed a map of the dust temperature so that the $100 \mu \mathrm{m}$ map may be converted to a map proportional to dust column density. The dust temperature varies from 17 to $21 \mathrm{~K}$, which is modest but does modify the estimate of the dust column by a factor of 5 . The result of these manipulations is a map with DIRBE quality calibration and IRAS resolution. A wealth of filamentary detail is apparent on many different scales at all Galactic latitudes. In high-latitude regions, the dust map correlates well with maps of $\mathrm{H} \mathrm{I}$ emission, but deviations are coherent in the sky and are especially conspicuous in regions of saturation of $\mathrm{H}$ I emission toward denser clouds and of formation of $\mathrm{H}_{2}$ in molecular clouds. In contrast, high-velocity $\mathrm{H}$ I clouds are deficient in dust emission, as expected.

To generate the full-sky dust maps, we must first remove zodiacal light contamination, as well as a possible cosmic infrared background (CIB). This is done via a regression analysis of the $100 \mu \mathrm{m}$ DIRBE map against the Leiden-Dwingeloo map of $\mathrm{H}_{\mathrm{I}}$ emission, with corrections for the zodiacal light via a suitable expansion of the DIRBE $25 \mu \mathrm{m}$ flux. This procedure removes virtually all traces of the zodiacal foreground. For the $100 \mu \mathrm{m}$ map no significant CIB is detected. At longer wavelengths, where the zodiacal contamination is weaker, we detect the CIB at surprisingly high flux levels of $32 \pm 13 \mathrm{nW} \mathrm{m} \mathrm{m} \mathrm{sr}^{-1}$ at $140 \mu \mathrm{m}$ and of $17 \pm 4 \mathrm{nW} \mathrm{m}{ }^{-2} \mathrm{sr}^{-1}$ at $240 \mu \mathrm{m}$ (95\% confidence). This integrated flux $\sim 2$ times that extrapolated from optical galaxies in the Hubble Deep Field.

The primary use of these maps is likely to be as a new estimator of Galactic extinction. To calibrate our maps, we assume a standard reddening law and use the colors of elliptical galaxies to measure the reddening per unit flux density of $100 \mu \mathrm{m}$ emission. We find consistent calibration using the $B-R$ color distribution of a sample of the 106 brightest cluster ellipticals, as well as a sample of 384 ellipticals with $B-V$ and $\mathrm{Mg}$ line strength measurements. For the latter sample, we use the correlation of intrinsic $B-V$ versus $\mathrm{Mg}_{2}$ index to tighten the power of the test greatly. We demonstrate that the new maps are twice as accurate as the older Burstein-Heiles reddening estimates in regions of low and moderate reddening. The maps are expected to be significantly more accurate in regions of high reddening. These dust maps will also be useful for estimating millimeter emission that contaminates cosmic microwave background radiation experiments and for estimating soft X-ray absorption. We describe how to access our maps readily for general use.
\end{abstract}

Subject headings: cosmic microwave background — diffuse radiation — dust, extinction infrared: ISM: continuum - interplanetary medium

\section{INTRODUCTION}

In the past 15 years two NASA missions have revolutionized our knowledge of the diffuse interstellar medium. The path-breaking Infrared Astronomy Satellite (IRAS) mission of 1983 led to the first full-sky maps of the diffuse background radiation in four broadband infrared channels, centered at $12,25,60$, and $100 \mu \mathrm{m}$, with a $\sim 5^{\prime}$ beam. The DIRBE experiment (Diffuse Infrared Background Experiment) on board the COBE satellite imaged the full sky in 10 broad photometric bands from 1 to $240 \mu \mathrm{m}$ with a beam of 0.7 . This experiment, for all but the shortest wavelength channels, was active for 42 weeks in 1989-1990 before its ${ }^{4} \mathrm{He}$ cryogen was exhausted. Although IRAS was optimized to detect point sources and sources of small angular extent (Beichman et al. 1988), it has been possible to create large-area sky maps from the IRAS data stream
(ISSA images; Wheelock et al. 1994). Striping artifacts from time variation in the zodiacal foreground emission have largely been filtered out of the individual ISSA maps, but artifacts remain and the zero point has large-scale drifts across the images. The DIRBE experiment had a much better control of the absolute calibration and has two further channels to the submillimeter than does IRAS (at 140 and $240 \mu \mathrm{m}$ ), but the maps have much lower angular resolution.

The diffuse emission seen in these experiments is a superposition of zodiacal foreground emission, dust and molecular emission from the interstellar medium (ISM), point sources from within and beyond the Milky Way, and possibly a diffuse extragalactic background. Separating these components has proven to be a most difficult task (Hauser 1996). The zodiacal emission dominates the 12 and $25 \mu \mathrm{m}$ channels and is a quite serious contaminant at $60 \mu \mathrm{m}$, while 
its contribution to the $100 \mu \mathrm{m}$ maps is less strong. Modeling of the zodiacal emission has proven quite complex (Reach et al. 1996). One must consider that the zodiacal dust in the ecliptic plane has a distribution of temperatures, that the tilt of Earth's orbit relative to the midplane of the dust is readily detectable, and that the zodiacal emission shows a strong dependence on solar elongation - information that is lost if one uses only the annual-averaged map of the DIRBE data.

A full-sky map of the diffuse radiation serves many purposes, and we here report our efforts to combine the $100 \mu \mathrm{m}$ maps of IRAS and DIRBE in a manner designed to be accessible to the general astrophysical community. To construct this map, we have removed the zodiacal foreground emission from the DIRBE maps, removed striping artifacts from the IRAS/ISSA maps, subtracted confirmed point sources, and combined the maps in such a way as to preserve the DIRBE calibration and IR $A S$ resolution. We have used the ratio of $100 \mu \mathrm{m}$ to $240 \mu \mathrm{m}$ emission to deduce a dust color temperature, allowing us to translate the $100 \mu \mathrm{m}$ emission to a column density of radiating dust. (A preliminary version of this work appeared in Schlegel 1995.) Such a procedure may be inadequate toward complex, dense clouds at low Galactic latitude, but in most directions the emission is dominated by dust within a single environment and radiation field.

Since the diffuse emission in the infrared is a direct measure of the column density of the interstellar dust, such a map can be used as a measure of extinction for extragalactic objects. As we shall argue below, the new dust map has better angular resolution and better control of systematics than possible with the reddening maps of Burstein \& Heiles (1978, 1982, hereafter BH). The essence of the Burstein-Heiles procedure was to assume that variations in the dust-to-neutral gas ratio can be adequately modeled by the smoothed mean background of galaxies (at northern declinations) or that this ratio is constant (at southern declinations). We make no assumptions about variations in the dust-to-gas ratio; neither do we need make any assumption concerning the correlation of diffuse ionized gas with neutral hydrogen. We only make the weaker assumption that the distribution of dust grain sizes is everywhere the same, since the relationship between UV/optical extinction and far-IR emission depends on the grain size distribution (Draine \& Lee 1984; Draine \& Anderson 1985; Guhathakurta \& Draine 1989; Mathis \& Cardelli 1992). The diffuse gas, where our results apply, is thought to have reasonably uniform dust properties, with values of $R_{V} \equiv$ $A(V) / E(B-V) \approx 3.1$. Since neither the Burstein-Heiles nor our assumptions are true for all environments, a comparison between the different methods is of interest.

To calibrate the extinction curve, we extensively explored the anticorrelation of counts in cells of the Automatic Plate Measuring Facility (APM) galaxy survey (Maddox et al. 1990a, 1990b) with $100 \mu \mathrm{m}$ emission, finding a consistent calibration in different directions of the sky. Due to complications in translating this calibration into $E(B-V)$, we shall defer this discussion to a separate paper (Finkbeiner et al. 1997).

Since we find that the $100 \mu \mathrm{m}$ maps are well correlated with $\mathrm{H}$ I emission at high latitudes, we do not expect the new extinction maps to deviate grossly from the BursteinHeiles reddening estimates when averaged to the same scales. But many dusty regions have filamentary structure with large fluctuations in extinction estimates over angular scales much smaller than the resolution of the $\mathrm{BH}$ maps. Furthermore, at low Galactic latitudes, regions such as Orion and Ophiuchus might be saturated in $\mathrm{H}$ I emission or partially ionized, whereas the dust remains optically thin.

Another important application for maps of the diffuse emission is comparison with cosmic microwave background radiation (CMBR) fluctuation maps. Recent analyses of the COBE/DMR fluctuations (Kogut et al. 1996) show a positive correlation with DIRBE maps, particularly in the North Polar Spur region. Similar correlations are seen between the Saskatoon CMBR maps and the IRAS maps (Oliveira et al. 1997; Leitch et al. 1997; Jaffe et al. 1997). This is unexpected, since these $40 \mathrm{GHz}$ CMBR experiments are not sensitive to dust emission, but are sensitive to free-free emission from the warm ionized medium (WIM; Gaustad, McCullough, \& Van Buren 1996; Simonetti, Dennison, \& Topsana 1996). This implies that the WIM is at least partly correlated with the cold dusty medium and that our dust maps could be used as a model for both components (Reynolds 1995). Alternatively, the observed CMBR correlations might be explained by small spinning dust grains producing continuum emission in the 10-100 GHz range (Draine \& Lazarian 1997), in which case the dust maps should correlate extremely well with CMBR observations over the entire sky.

This paper is organized as follows: Section 2 describes the processing of the DIRBE data. How the weekly averaged DIRBE maps are trimmed and then reconstituted into annual-average maps that are more suitable for our simple treatment of the zodiacal light removal. We fit a zodiacal light model by maximizing the correlation between the DIRBE maps and $\mathrm{H}_{\mathrm{I}}$ in regions of high Galactic latitude and low flux.

The diffuse cirrus emission is expected to have a reasonably uniform temperature, since the ultraviolet radiation field that is heating the dust must be a smooth function of angular position, except within dense clouds. Fortunately, the emission at 100 and $240 \mu \mathrm{m}$ is expected to be dominated by grains sufficiently large to be in equilibrium with the radiation field (e.g., Draine \& Anderson 1985; Guhathakurta \& Draine 1989), so that the $100 \mu \mathrm{m} / 240 \mu \mathrm{m}$ color ratio can provide a useful measure of grain temperature. In $\S 2.4$ we describe how we measure the temperature in the presence of substantial noise in the $240 \mu \mathrm{m}$ map. This information is used to translate the $100 \mu \mathrm{m}$ emission to dust column density.

The inclusion of the IRAS data is discussed in $\S 3$. Before we can combine the IRAS/ISSA map with the DIRBE map, we must first deal with the ISSA boundaries and discontinuities. The ISSA maps are destriped versions of the original $I R A S$ scans, but further destriping is possible and essential for high angular resolution studies. We use a matched set of window functions that low-pass filter the DIRBE map to $1^{\circ}$ resolution and high-pass filter IRAS/ISSA maps on the same scale. The combined map is then simply the sum of these two filtered maps. Section 4 details the removal of extragalactic objects and point sources from the maps, leaving behind only a map of the diffuse dust emission.

In $\S 5$ we present a simple procedure for normalizing the reddening per unit flux density of dust emission. We correlate the residual of the $B-V$ versus $\mathrm{Mg}_{2}$ index for 389 elliptical galaxies against the estimated reddening and adjust the normalization until the slope of the relation is zero. The resulting calibration has an uncertainty of only $10 \%$. We also show that the maps have an accuracy of $16 \%$ 
in predicting reddening, which is twice as good as the older BH procedure.

A summary of all these reprocessing procedures in presented in $\S 6$. Readers uninterested in the details of the analysis should skip directly to this point. General discussion and conclusions are presented in $\S \S 7$ and 8 . Appendix A discusses the contamination level of stars that remain in the maps. Appendix B describes how reddenings and extinction in different optical and infrared filters can be related. Appendix $\mathrm{C}$ describes how the final maps can be accessed.

\section{DUST COLUMN DENSITIES FROM THE DIRBE MAPS}

\subsection{The DIRBE Data Set}

The DIRBE data set is in the public domain and was obtained from the National Space Science Data Center (NSSDC) on CD-ROM. ${ }^{1}$ For a complete description of the DIRBE instrument, see Boggess et al. (1992). Both Annual Average Skymaps and maps averaged over each week of the mission are provided. The data are binned to 393,216 0.32 $\times 0.32$ pixels on the $C O B E$ Quadrilateralized Spherical Cube. (For a description of this "skycube" projection, see Chan \& O'Neill 1975.) As described below, we create new annual-average 25 and $100 \mu \mathrm{m}$ skymaps from the 41 weekly maps in skycube format in which the weighting function of each week is forced to be the same for both channels. We then reproject the data to standard equal-area polar projections oversampled to 512 pixels from $b=0^{\circ}$ to $90^{\circ}(0.0250$ $\operatorname{deg}^{2}$ pixel $\left.^{-1}\right)$.

In order to preserve total flux to a good approximation, we initially projected to a Lambert (polar) mapping with $4 \times 4$ smaller pixels that were then binned to the 0.0250 $\mathrm{deg}^{2}$ resolution. (Note that the surface brightness of the cirrus is preserved in this reprojection.) These maps for each passband $b$ are denoted by $D_{b}$, and are in units of $\mathrm{MJy} \mathrm{sr}^{-1}$. We use the $25 \mu \mathrm{m}$ map as a model of the zodiacal light and the 100 and $240 \mu \mathrm{m}$ maps for measuring the temperature and column density of the dust.

\subsection{Zodiacal Light Removal}

The DIRBE maps are severely contaminated by zodiacal emission from dust in the solar system, otherwise known as the IPD (interplanetary dust). The IPD primarily reradiates thermally at $\sim 280 \mathrm{~K}$ in the infrared (for $v^{0}$ emissivity). Its emission per unit column density is substantially higher than that of $20 \mathrm{~K}$ Galactic dust: using an $\alpha=2$ emissivity law, the $100 \mu \mathrm{m}$ emission of the IPD is larger by a factor $\sim 10^{5}$ than that of Galactic dust for an equivalent column density. The brightest zodiacal emission is in the ecliptic plane, at the level of $\sim 10 \mathrm{MJy} \mathrm{sr}^{-1}$. Based upon our solutions, this corresponds to an extinction at the negligible level of $A(B) \approx 10^{-6}$ mag. Thus, the removal of zodiacal light emission is critical, as it makes no measurable contribution to optical extinction.

The $25 \mu \mathrm{m}$ maps, where the IPD emission peaks, are used to model the IPD contamination at the longer wavelengths. Before removing this contamination, the DIRBE annualaverage data must first be regenerated so that the data at all wavelengths are sampled at the same time and through the same IPD.

\footnotetext{
${ }^{1}$ Accessible via the World Wide Web at http:// nssdc.gsfc.nasa.gov/astro/astro_home.html.
}

The zero points of the zodiacal contamination are constrained by $\mathrm{H}$ I maps at high Galactic latitude.

\subsubsection{Dependence on Time and Solar Elongation Angle}

Removal of the zodiacal light is a difficult problem. The observing strategy of the $C O B E$ satellite resulted in a lower signal-to-noise ratio in the ecliptic plane where the zodiacal light is most prominent. The number of high-quality DIRBE observations per pixel ranged from $\sim 200$ at the ecliptic equator to $\sim 800$ near the ecliptic poles. The leastsampled regions were approximately cones of $90^{\circ}$ opening angle centered at ecliptic $(\lambda, \beta)=\left(100^{\circ}, 0^{\circ}\right)$ and $(\lambda, \beta)=$ $\left(280^{\circ}, 0^{\circ}\right)$. The DIRBE maps exhibit a striping pattern perpendicular to the ecliptic plane; scans adjacent in the sky correspond to temporally separated instrument tracks, taken at varying solar elongation angles, through different column densities of IPD.

Measurements for each week of the mission were used to construct weekly SKYMAPS. The DIRBE Annual Average Skymaps are a combination of the weekly maps, averaged by the number of times that each pixel was observed in a weekly map. Unfortunately, the whole sky is not scanned each week, and some parts of the scanned region are omitted in certain weeks as a result of the interference from the Moon and planets. Furthermore, not all the detectors were on at all times.

A cursory glance at the weekly skymaps reveals a serious problem of combining data in this way. Near the ecliptic plane the $25 \mu \mathrm{m}$ flux exhibits a strong dependence on solar elongation angle $e$. During the 41 weeks, data were typically taken between $e=64^{\circ}$ and $124^{\circ}$, and the $25 \mu \mathrm{m}$ flux varies by a factor of 2 over this range. Comparison with the 100 $\mu \mathrm{m}$ IPD flux also reveals a strong temperature gradient with respect to $e$. This is easily explained by assuming a radial gradient of IPD density from the Sun, because a line of sight passing close to the Sun must traverse more (and hotter) dust than a line of sight at larger solar elongation. As a further complication, because the 10 passbands were not always observed simultaneously, the weekly skymaps of the weighting function do not always match across all channels. Contributions to the Annual Average Skymaps were weighted according to the number of observations made in each channel, in spite of the strong dependence on elongation angle.

In cases where the measurement noise is overwhelmed by the time-dependent systematic variations, this method of averaging is inappropriate. The weights assigned to sky pixels are thus not always the same for the 25 and $100 \mu \mathrm{m}$ weekly maps, leading to weighted Annual Average Skymaps that cannot be directly compared to each other. To rectify this, we recombine the 41 weekly skymaps using the same weight for the same pixels in different passbands. We use the error maps for the $100 \mu \mathrm{m}$ passband as weights in all other passbands. Because the IPD temperature gradient is strongest at low $e$, we further delete all data in each weekly map taken at $e<80^{\circ}$. The resulting maps have noise and artifacts that are somewhat worse than the Annual Average Skymaps from the NSSDC; however, the artifacts are the same at both wavelengths. The resulting $25 \mu \mathrm{m}$ map is appropriate for modeling the IPD contamination at other wavelengths.

\subsubsection{Scale and Zero Point of the Zodiacal Light}

The spatial-temporal variations of zodiacal light are complicated and difficult to model analytically. Our pro- 
cedure for removing the zodiacal light assumes that the 100 $\mu \mathrm{m}$ map correlates linearly with $\mathrm{H}_{\mathrm{I}}$ at high Galactic latitudes and low flux levels (Boulanger et al. 1996). Note that one should also expect some dust within the extensive ionized $\mathrm{H}^{+}$zones and that the column density ratio $\mathrm{N}\left(\mathrm{H}^{+}\right)$/ $N(\mathrm{H}$ I) has considerable scatter in different directions (Reynolds 1990). The relationship between the diffuse ionized and neutral hydrogen in any direction is complex (Reynolds 1995); some of the $\mathrm{H}^{+}$is associated with neutral gas, while other ionized clouds adjoin neutral regions. We are, of course, interested in measuring the dust associated with both components, and to the degree that there is dust within $\mathrm{H}^{+}$regions not associated with neutral regions, the scatter of dust and $\mathrm{H}$ I will be increased. The degree of scatter between neutral gas and dust is therefore of considerable interest, but this scatter should not bias our procedure.

We consider the $25 \mu \mathrm{m}$ map as a template of the unwanted zodiacal foreground, as the $25 \mu \mathrm{m}$ channel is so thoroughly dominated by the IPD. In a few regions where the Galactic cirrus contributes more than a few percent to the $25 \mu \mathrm{m}$ emission, we interpolate the $25 \mu \mathrm{m}$ map in bins of constant ecliptic latitude. To first order, we subtract scaled versions of the $25 \mu \mathrm{m}$ map in order to minimize the scatter between the $\mathrm{H}_{\mathrm{I}}$ and zodiacal-corrected 100 and $240 \mu \mathrm{m}$ emission. We further refine this correction with the addition of a quadratic term to account for temperature variations in the IPD.

Fitting blackbody functions to the zodiacal light from 12 to $60 \mu \mathrm{m}$, we find that the color temperature does not vary by more than $10 \%$ from ecliptic equator to the poles. This allows us to make a first-order correction under the assumption that the IPD is at a constant temperature. The DIRBE $25 \mu \mathrm{m}$ map, $D_{25}$, is directly scaled to model the zodiacal contamination at longer wavelengths. The raw maps for these other DIRBE passbands, $D_{b}$, are corrected to

$$
D_{b}^{L}=D_{b}-A_{b} D_{25}-B_{b} \text {. }
$$

The coefficient $A_{b}$ determines the scaling of the zodiacal emission from the $25 \mu \mathrm{m}$ DIRBE passband to passband $b$. The zero-point offset $B_{b}$ is used to account for a multitude of nonzodiacal contributions to the 25 and $100 \mu \mathrm{m}$ maps, either from the Galaxy or from extragalactic light. Although the $25 \mu \mathrm{m}$ map is relatively free of point sources, the 10 brightest sources have been masked to their local averages (see Table 1). Note that these sources were not excluded from the 100 or $240 \mu \mathrm{m}$ maps.

Assuming that $\mathrm{H}$ I correlates linearly with dust at high latitudes and low flux levels, we make use of the $\mathrm{H}$ i flux to determine the zodiacal flux scaling coefficients and zeropoint offsets. We compare the emission $D^{L}$ to the $\mathrm{H}$ I column density $H$ from the Leiden-Dwingeloo $21 \mathrm{~cm}$ survey (Hartmann \& Burton 1996). The $21 \mathrm{~cm}$ emission is summed in the velocity range $-72<v_{\mathrm{LSR}}<+25 \mathrm{~km} \mathrm{~s}^{-1}$, which samples almost all of the Galactic $H_{\text {I }}$ at high Galactic latitudes. (At high latitudes, $\mathrm{H}$ I outside of this range shows no correlation with the $100 \mu \mathrm{m}$ maps.) We solve for the coefficients $A_{b}$ and $B_{b}$ of equation (1) and the $\mathrm{H}$ I-toDIRBE ratio $\zeta_{b}$ using a least-squares minimization of

$$
\chi^{2}=\sum_{i}\left[\frac{\zeta_{b}\left(D_{b}^{L}\right)_{i}-H_{i}}{\sigma_{i}}\right]^{2}
$$

TABLE 1

\begin{tabular}{|c|c|c|c|c|c|c|}
\hline $\begin{array}{c}l \\
(\mathrm{deg})\end{array}$ & $\begin{array}{c}b \\
(\mathrm{deg})\end{array}$ & $D_{25}$ & $X$ & $S$ & $\begin{array}{l}f_{60} \\
\text { (Jy) }\end{array}$ & Source \\
\hline 4.6 & 35.5 & & $\mathrm{X}$ & & & \\
\hline 5.2 & 36.7 & & $\mathrm{X}$ & & & \\
\hline 28.8 & 3.5 & & & $\mathrm{X}$ & 3041 & IRAS $18288-0207$ \\
\hline 49.4 & -0.3 & & & $\mathrm{X}$ & 5263 & IRAS $19209+1421$ \\
\hline 80.1 & -6.4 & $\mathrm{X}$ & & & & \\
\hline 90.7 & -46.5 & & & $\mathrm{X}$ & & \\
\hline 94.4 & -5.3 & & & $\mathrm{X}$ & & \\
\hline 95.5 & -87.5 & & $\mathrm{X}$ & $\mathrm{X}$ & & \\
\hline 105.0 & 68.8 & & $\mathrm{X}$ & & & \\
\hline 106.8 & 5.3 & & & $\mathrm{X}$ & 11230 & IRAS $22176+6303$ \\
\hline 109.9 & 2.1 & & & $\mathrm{X}$ & 12830 & IRAS $22540+6146$ \\
\hline 111.5 & 0.8 & & & $\mathrm{X}$ & 7073 & IRAS $23113+6113$ \\
\hline 118.1 & 5.0 & & & $\mathrm{X}$ & & \\
\hline 121.3 & -21.5 & & $\mathrm{X}$ & & & M31 \\
\hline 123.1 & -6.4 & & & $\mathrm{X}$ & & \\
\hline 126.6 & -0.7 & & & $\mathrm{X}$ & & \\
\hline 133.5 & -31.3 & & $\mathrm{X}$ & & & \\
\hline 133.7 & 1.2 & & & $\mathrm{X}$ & 12000 & IRAS $02219+6152$ \\
\hline 141.4 & 40.6 & & $\mathrm{X}$ & $\mathrm{X}$ & 1170 & IRAS $09517+6954$ \\
\hline 165.4 & -9.0 & & & $\mathrm{X}$ & 3121 & IRAS $04269+3510$ \\
\hline 168.0 & -58.0 & $\mathrm{X}$ & & & & \\
\hline 173.7 & 2.7 & & & $\mathrm{X}$ & 1709 & IRAS $05375+3540$ \\
\hline 178.0 & -31.5 & $\mathrm{X}$ & & & & \\
\hline 187.0 & 1.3 & $\mathrm{X}$ & & & & \\
\hline 189.9 & 0.5 & & & $\mathrm{X}$ & 1157 & IRAS $06063+2040$ \\
\hline 197.8 & 56.0 & $\mathrm{X}$ & & & & \\
\hline 205.3 & -14.4 & & & $\mathrm{X}$ & 1291 & IRAS $05445+0016$ \\
\hline 206.6 & -16.3 & & & $\mathrm{X}$ & 7894 & IRAS $05393-0156$ \\
\hline 209.0 & 19.6 & $\mathrm{X}$ & & & & \\
\hline 213.7 & -12.5 & & & $\mathrm{X}$ & 13070 & IRAS $06056-0621$ \\
\hline 221.4 & 45.1 & $\mathrm{X}$ & $\mathrm{X}$ & & 5652 & IRAS $09452+1330$ \\
\hline 239.4 & -5.1 & $\mathrm{X}$ & & & 1453 & IRAS $07209-2540$ \\
\hline 243.2 & 0.3 & & & $\mathrm{X}$ & & \\
\hline 265.1 & 1.4 & & & $\mathrm{X}$ & 8333 & IRAS $08576-4334$ \\
\hline 272.7 & -39.5 & $\mathrm{X}$ & & & & \\
\hline 274.0 & -1.1 & & & $\mathrm{X}$ & 7500 & IRAS $09227-5146$ \\
\hline 279.3 & -33.3 & & $\mathrm{X}$ & & & LMC \\
\hline 287.6 & -0.6 & & & $\mathrm{X}$ & 13470 & IRAS $10431-5925$ \\
\hline 302.5 & -44.4 & & $\mathrm{X}$ & & & SMC \\
\hline 305.4 & 0.2 & & & $\mathrm{X}$ & 8861 & IRAS $13092-6218$ \\
\hline 309.3 & 19.2 & & $\mathrm{X}$ & & & \\
\hline 316.5 & 21.0 & & $\mathrm{X}$ & & & \\
\hline 318.0 & 32.9 & $\mathrm{X}$ & & & & \\
\hline 353.1 & 16.9 & & & $\mathrm{X}$ & 2196 & IRAS $16235-2416$ \\
\hline 359.9 & -17.7 & & $\mathrm{X}$ & $\mathrm{X}$ & & \\
\hline
\end{tabular}

MASKED SOURCES IN DIRBE MAPS

NotE.-List of sources removed from the $D_{25}$ map used to construct the zodiacal light model, the temperature correction $X$ map, or the $\mathscr{S}$ map used to combine the DIRBE and IRAS $100 \mu \mathrm{m}$ maps. Sources removed from each map are denoted by an "X." Those that correspond to a bright source in the IRAS Point Source Catalog have their $60 \mu \mathrm{m}$ flux and name listed.

in each passband. The errors in this relation are systematic and difficult to express analytically. Therefore, we take the uncertainties $\sigma_{i}$ to be proportional to the $H_{\text {I flux. The }}$ fitting procedure is performed on the maps binned to 2.5 and limited to those areas of the sky with low emission, $\mathrm{H} \mathrm{I}<200 \mathrm{~K} \mathrm{~km} \mathrm{~s}^{-1}$ or $N(\mathrm{H})<3.7 \times 10^{18} \mathrm{~cm}^{-2}(19 \%$ of the sky). Two dust clouds with unusual temperature and the region immediately around the very bright source NGC 253 are omitted from the fits. The results of these fits for $b=100,140$, and $240 \mu \mathrm{m}$ are presented in Table 2 , and a scatter diagram is shown in Figure 1 both $(a)$ before and $(b)$ after the correction.

Temperature variations in the IPD make the linear correction of equation (1) inadequate for our purposes. Based on $12 \mu \mathrm{m} / 25 \mu \mathrm{m}$ and $25 \mu \mathrm{m} / 60 \mu \mathrm{m}$ ratios, the color tem- 
TABLE 2

ZODIACAL LIGHT MODEL COEFFICIENTS

\begin{tabular}{|c|c|c|c|c|c|c|c|}
\hline $\begin{array}{c}\lambda \\
(\mu \mathrm{m})\end{array}$ & Fit & $A$ & $B$ & $Q$ & $\begin{array}{c}D / \mathrm{Gas} \\
\left(\mathrm{MJy} \mathrm{sr}^{-1}\right) /(\mathrm{K} \mathrm{km} / \mathrm{s})\end{array}$ & $\begin{array}{l}\text { Scatter } \\
(\%)\end{array}$ & $\begin{array}{c}Z \\
\left(\mathrm{MJy} \mathrm{sr}^{-1}\right)\end{array}$ \\
\hline $100 \ldots \ldots$ & $\mathrm{L}$ & $0.1607 \pm 0.0005$ & $-0.958 \pm 0.015$ & ; & $0.01363 \pm 0.00012$ & 19 & $2.383 \pm .014$ \\
\hline $140 \ldots \ldots$ & $\mathrm{L}$ & $0.0738 \pm 0.0005$ & $0.058 \pm 0.015$ & $\ldots$ & $0.02555 \pm 0.00012$ & 21 & $1.590 \pm .032$ \\
\hline $240 \ldots \ldots$ & $\mathrm{L}$ & $0.0192 \pm 0.0005$ & $0.668 \pm 0.015$ & $\ldots$ & $0.01895 \pm 0.00012$ & 18 & $1.068 \pm .023$ \\
\hline $100 \ldots \ldots$ & $\mathrm{Q}$ & $0.0810 \pm 0.0020$ & $0.623 \pm 0.043$ & $8.79 E-4 \pm 2.2 E-5$ & $0.01222 \pm 0.00012$ & 16 & $2.697 \pm .014$ \\
\hline $140 \ldots \ldots$ & $\mathrm{Q}$ & $-0.0653 \pm 0.0020$ & $2.82 \pm 0.043$ & $1.53 \mathrm{E}-3 \pm 2.2 \mathrm{E}-5$ & $0.02309 \pm 0.00012$ & 19 & $2.132 \pm .032$ \\
\hline $240 \ldots$ & $\mathrm{Q}$ & $-0.0504 \pm 0.0020$ & $2.05 \pm 0.043$ & $7.68 \mathrm{E}-4 \pm 2.2 \mathrm{E}-5$ & $0.01772 \pm 0.00012$ & 17 & $1.338 \pm .023$ \\
\hline
\end{tabular}

Note.- $A, B$, and $Q$ are the best-fit coefficients for the linear $(\mathrm{L})$ or quadratic $(\mathrm{Q})$ zodiacal light model. The ratio between the modelcorrected IR emission and $\mathrm{H}$ I emission, $D / G$ as, has the quoted scatter in the range $[0,200] \mathrm{K} \mathrm{km} \mathrm{s}^{-1}$. After removing the contribution from dust, $Z$ is the zodiacal light plus the CIB averaged at high ecliptic latitudes $\left(\beta>85^{\circ}\right)$.

perature of the IPD varies by $5 \%$ for an $\alpha=1$ emissivity law or by $10 \%$ for an $\alpha=2$ emissivity law. Because the 100 $\mu \mathrm{m}$ band is on the Rayleigh-Jeans side of the spectrum, this results in a $5 \%$ or $10 \%$ error in zodiacal light. As the zodiacal light contributes up to $10 \mathrm{MJy} \mathrm{sr}^{-1}$ at $100 \mu \mathrm{m}$ in the ecliptic plane, this results in absolute errors of $1 \mathrm{MJy} \mathrm{sr}^{-1}$, with a strong ecliptic latitude dependence. A constanttemperature linear model of zodiacal dust removal is a good first approximation, but it is clearly too simplistic. Because the neighboring passbands at 12 and $60 \mu \mathrm{m}$ are a complex mix of zodiacal and nonzodiacal contributions, it is desir-

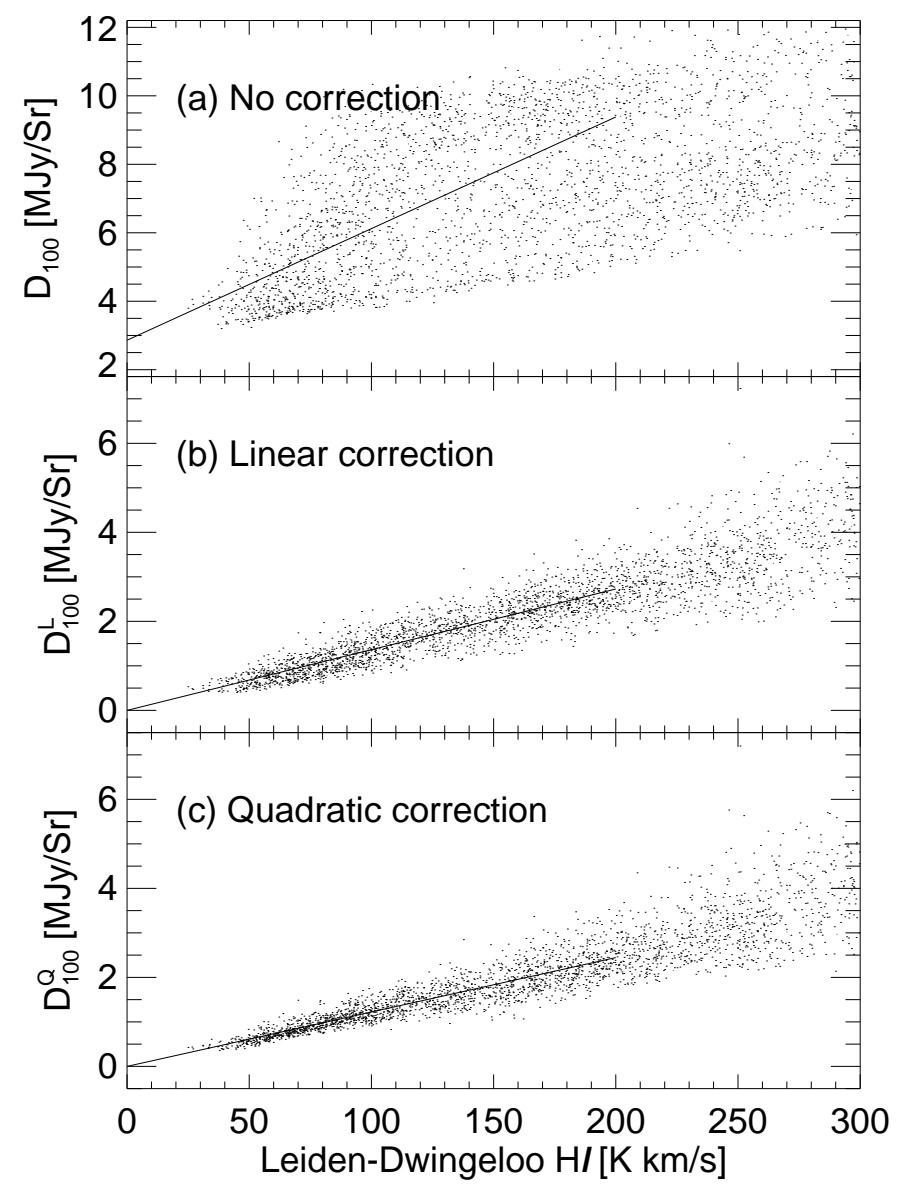

FIG. 1.-The $100 \mu \mathrm{m}-\mathrm{H}$ I correlation with (a) no correction, (b) linear correction, and (c) quadratic correction for zodiacal contamination. The fits in the range $[0,200] \mathrm{K} \mathrm{km} \mathrm{s}^{-1}$ are shown as solid lines. able to model the variation in IPD temperature using only the $25 \mu \mathrm{m}$ map, rather than some linear combination of passbands. Thus, we are driven to higher order fits using only the $25 \mu$ m map.

The temperature variations in the IPD are most strongly a function of ecliptic latitude $\beta$. An adequate second-order function for modeling the variations in IPD emission at longer wavelengths includes a term that is linear in the product of $D_{25}$ and $\bar{D}_{25}(\beta)$, the mean $25 \mu$ m emission at each ecliptic latitude (limited to $|b|>20^{\circ}$ to minimize Galactic contamination in the model). With a scaling coefficient $Q_{b}$, the corrected maps then become

$$
D_{b}^{Q}=D_{b}-\left[A_{b}+Q_{b} \bar{D}_{25}(\beta)\right] D_{25}-B_{b} .
$$

The above is referred to as the quadratic correction. Solutions are again found via equation (2) using the same region of the sky as before. Results are presented in Table 2. Since the linear and quadratic contributions are not orthogonal, there is no particular physical meaning to the coefficients derived in the quadratic fits; they are merely the coefficients that yield the minimum $\chi^{2}$.

The result of the quadratic correction is shown in Figure $1 c$. At $100 \mu \mathrm{m}$ the quadratic correction is clearly superior to the linear correction, reducing the rms scatter from $19 \%$ to $16 \%$ (for $\mathrm{H} \mathrm{I}<200 \mathrm{~K} \mathrm{~km} \mathrm{~s}^{-1}$ ). At $240 \mu \mathrm{m}$ the difference between the two fits is negligible. This is both because the $240 \mu \mathrm{m}$ map has a higher inherent noise and because the zodiacal light correction at such long wavelengths is smaller. Even though there is no obvious difference in fitting methods at $240 \mu \mathrm{m}$, we apply the quadratic correction to both passbands in order to avoid introducing systematic errors into the determination of the dust temperature. Using the Bell Laboratories H I survey (Stark et al. 1992) instead of Leiden-Dwingeloo results in a zero-point change in the final $100 \mu \mathrm{m}$ map of only $0.013 \mathrm{MJy} \mathrm{sr}^{-1}$, with a standard deviation of $0.047 \mathrm{MJy} \mathrm{sr}^{-1}$.

In principle, the fit should be made between the $\mathrm{H}$ I gas and the total column density of dust. The total column is a complicated function of the 100 and $240 \mu \mathrm{m}$ maps, and the zodiacal light coefficients for both those maps need be fitted simultaneously. This nonlinear fit with six parameters is not stable, primarily because the DIRBE $240 \mu \mathrm{m}$ signal-tonoise ratio is too low at high latitudes. For this reason, we have performed the fit independently for the 100 and 240 $\mu \mathrm{m}$ maps. As long as the temperature of the dust does not vary significantly and systematically with ecliptic latitude, then the fit coefficients for our zodiacal model should be valid. As we discuss in $\S 2.3$, variations in the Galactic dust 
temperature at the high latitudes used in these fits are hardly measurable above the noise.

This least-squares minimization procedure is used only to remove the zodiacal foreground. Our measure of the best removal is minimal scatter between the far-IR dust emission and $21 \mathrm{~cm}$ emission. There could well be an additional component of dust that is not correlated with the $\mathrm{H}$ I emission, such as dust associated with diffuse $\mathrm{H}^{+}$regions. In such a situation, our procedure for zodiacal foreground removal should remain unbiased, but the interpretation of the fit coefficients would be different. The slope $A_{b}$ would signify the amount of dust associated with neutral gas mixed with ionized gas, while the offset $B_{b}$ would be the dust associated with instrumental offset, the cosmic infrared background (CIB), and dust emission associated with a uniform sphere of diffuse $\mathrm{H}^{+}$.

This method of zodiacal light correction is by no means definitive. A more thorough examination of the problem is currently under way by the DIRBE team (Kelsall et al. 1998). They are performing a detailed modeling of the IPD in three-dimensional space that includes the dependence of dust temperature and density on the distance from the Sun, as well as specific dust bands, and the inclination of the dust cloud with respect to the Earth's orbit. However, our model is adequate for the present analysis. In the worst case, assuming that all of the residual scatter in the corrected 100 $\mu \mathrm{m}$ plots at $\mathrm{H} \mathrm{I}<100 \mathrm{~K} \mathrm{~km} \mathrm{~s}^{-1}$ is caused by imperfectly subtracted zodiacal light, the rms of this error is $0.05 \mathrm{MJy}$ $\mathrm{sr}^{-1}$. This corresponds to an error of $0.004 \mathrm{mag}$ in $A(B)$.

\subsection{Zodiacal Removal to Isolate the CIB}

As a digression at this point, the question of the CIB was a major motivating factor for the DIRBE experiment. The extraction of the CIB has proven to be a considerable challenge (Hauser 1996), and to date, CIB detections from DIRBE data have been reported as upper limits. The regression analysis described above is one method to approach such a measurement, provided that the zodiacal model is adequate. The coefficient $B$ of the regression fits is a constant term that remains after the zodiacal foreground dust and all Galactic dust correlated with $\mathrm{H}$ I emission is removed. This term is not likely to be an instrumental offset, since DIRBE had a cold-load internal chopper, and the entire telescope was cold. A significant detection of $B$ is either a measure of the CIB or a result of some unknown instrumental artifact.

Since the zodiacal dust is confined to a thin zone in the ecliptic plane of the solar system, along directions away from the ecliptic plane, all the zodiacal foreground is approximately $1 \mathrm{AU}$ from the Sun. It is therefore reasonable to assume the zodiacal foreground emission of our reconstructed yearly maps to be the same temperature in all directions for sufficiently high $|\beta|$. Thus, a linear model should be adequate for removal of the zodiacal emission. However, one cannot look strictly at the ecliptic poles, since the $25 \mu \mathrm{m}$ maps vary only by a factor of 3.5 from the ecliptic plane to the pole; a suitable lever arm in $\beta$ is therefore essential for decoupling the isotropic CIB from the smoothly varying zodiacal foreground.

We have performed linear regression analyses for the 100 , 140 , and $240 \mu \mathrm{m}$ maps as a function of the lower limit on ecliptic latitude $|\beta|$ using the procedures described in $\S$ 2.2.2. The results are listed in Table 3. The quoted errors represent the formal $95 \%$ confidence intervals and do not
TABLE 3

CIB AT 100, 140, AND $240 \mu \mathrm{m}$

\begin{tabular}{cccc}
\hline \hline \multirow{2}{*}{$\begin{array}{c}\beta \mid \text { CUT } \\
(\mathrm{deg})\end{array}$} & \multicolumn{3}{c}{ FLUx $\left(\mathrm{MJy} \mathrm{sr}^{-1}\right)$} \\
\cline { 2 - 4 } & $100 \mu \mathrm{m}$ & $140 \mu \mathrm{m}$ & $240 \mu \mathrm{m}$ \\
\hline $0 \ldots \ldots \ldots$ & $-0.96 \pm 0.04$ & $0.06 \pm 0.08$ & $0.67 \pm 0.05$ \\
$5 \ldots \ldots \ldots$ & $-0.86 \pm 0.04$ & $0.28 \pm 0.09$ & $0.77 \pm 0.06$ \\
$10 \ldots \ldots$ & $-0.71 \pm 0.04$ & $0.55 \pm 0.09$ & $0.92 \pm 0.06$ \\
$15 \ldots \ldots$. & $-0.49 \pm 0.05$ & $0.87 \pm 0.10$ & $1.07 \pm 0.07$ \\
$20 \ldots \ldots$ & $-0.29 \pm 0.05$ & $1.13 \pm 0.13$ & $1.20 \pm 0.08$ \\
$30 \ldots \ldots$ & $0.02 \pm 0.08$ & $1.49 \pm 0.20$ & $1.34 \pm 0.14$ \\
$45 \ldots \ldots$ & $0.15 \pm 0.19$ & $1.69 \pm 0.50$ & $1.27 \pm 0.33$ \\
\hline
\end{tabular}

NoTE.-Derived CIB level using different cuts in ecliptic latitude for our zodiacal model. Errors denote $95 \%$ confidence limits, excluding systematic errors. The preferred solution uses the cut at $|\beta|=30^{\circ}$, as described in the text.

incorporate systematic errors caused by our choice of zodiacal model. For the zero-point term $B$, the quoted errors are dominated by covariance with the zodiacal coefficient $A$. At $100 \mu \mathrm{m}$ we have no significant detection, largely because the zodiacal foreground is quite substantial. At 140 and $240 \mu \mathrm{m}$ the determination of $B$ is significant and nonzero. These are possibly measurements of the CIB, as we discuss in $\S 7.4$ below.

\subsection{DIRBE Temperature Maps}

The main component of Galactic dust detected by DIRBE spans a color temperature range of 17-21 K for an assumed $\lambda^{-2}$ emissivity (Reach et al. 1995). At the extremes of this temperature range, the emission at $100 \mu \mathrm{m}$ will differ by a factor of 5 for the same column density of dust. Therefore, it is crucial to use temperature information to recover the dust column density. There are three useful DIRBE bands for computing the Galactic dust temperature, centered at 100, 140, and $240 \mu \mathrm{m}$. The median signal-to-noise ratio per pixel for these maps at $|b|>10^{\circ}$ is $164,4.2$, and 4.9 , respectively. Because of the high $\mathrm{S} / \mathrm{N}$ at $100 \mu \mathrm{m}$, we will use that map as a spatial template and multiply it by a temperature correction factor derived from the $100 \mu \mathrm{m} / 240$ $\mu \mathrm{m}$ ratio. The $140 \mu \mathrm{m}$ map is not used because of its large noise level compared to the nearby $100 \mu \mathrm{m}$ map. We attempt no temperature correction in regions of very low emission, since our procedure will introduce noise, and the exact temperature of the dust in these dilute regions is less important.

\subsubsection{FIR Spectrum of the Dust}

Estimation of the dust color temperature and column density is complicated by our lack of knowledge about dust opacities and temperature distributions. Our procedure works only if the large grains responsible for the emission at $100-240 \mu \mathrm{m}$ are in thermal equilibrium with the interstellar radiation field, and the smaller, transiently heated dust grains make a negligible contribution to emission at these wavelengths. Fortunately, these assumptions appear to be in agreement with our current understanding of interstellar dust emission (Guhathakurta \& Draine 1989; Sodroski et al. 1997).

Because the ISM is optically thin in the FIR, the intensity $I_{v}$ is given by

$$
I_{v}=\int d s \rho \kappa_{v} B_{v}(T)
$$


where $T$ is the grain temperature, $\rho$ is the mass density, $B_{v}$ is the Planck function, $\kappa_{v}$ is the opacity, and the integral is over the physical path length. Since the dust particles are small (radii $a<0.25 \mu \mathrm{m}$ ) compared to FIR wavelengths, the opacity $\kappa_{v}$ does not depend upon the details of the particle size distribution. However, the opacity does depend upon the nature of the material. In general, the opacity follows a power law in the FIR,

$$
\kappa_{v} \propto v^{\alpha}
$$

The Draine \& Lee (1984) model predicts that the FIR emission is predominately from graphite with $\alpha \approx 2.0$. Layerlattice materials, such as amorphous carbon, have opacities $\alpha \approx 1.0$, and silicates have $\alpha \approx 1.5$. We have chosen $\alpha=2.0$ in deriving our dust temperatures. Though this choice directly affects the temperature estimation, we show that our final dust column densities are completely insensitive to it. The reason for this insensitivity is that the DIRBE passbands are sufficiently near the Wien tail of the dust spectrum.

"Classical" grains with radii $a>0.05 \mu \mathrm{m}$ exposed to ambient starlight retain an equilibrium temperature. In $\S 2.4 .2$ we assume such an equilibrium and fit each line of sight with a single color temperature. However, each line of sight through the Galaxy may pass through several regions at different equilibrium temperatures. By ignoring multiple temperature components, we systematically underestimate the true column density. We have modeled this effect by fitting a single color temperature to emission from $18 \mathrm{~K}$ dust added to emission from another region at temperature $T_{B}$. For different choices of $T_{B}$ and its mass fraction $f_{B}$, we compare the recovered dust column to the true column density (Fig. 2). For the range $15<T_{B}<21.5 \mathrm{~K}$, the true column is at most underestimated by $10 \%$. A factor of 2 error would result only in contrived circumstances with $T_{B}<12 \mathrm{~K}$ or $T_{B}>33 \mathrm{~K}$. As the majority of the sky spans only a $5^{\circ}$ range of temperature, our single-temperature model is deemed satisfactory.

Another constituent of the dust that we have ignored is very small grains (VSGs), with radii $a \lesssim 0.005 \mu \mathrm{m}$. These

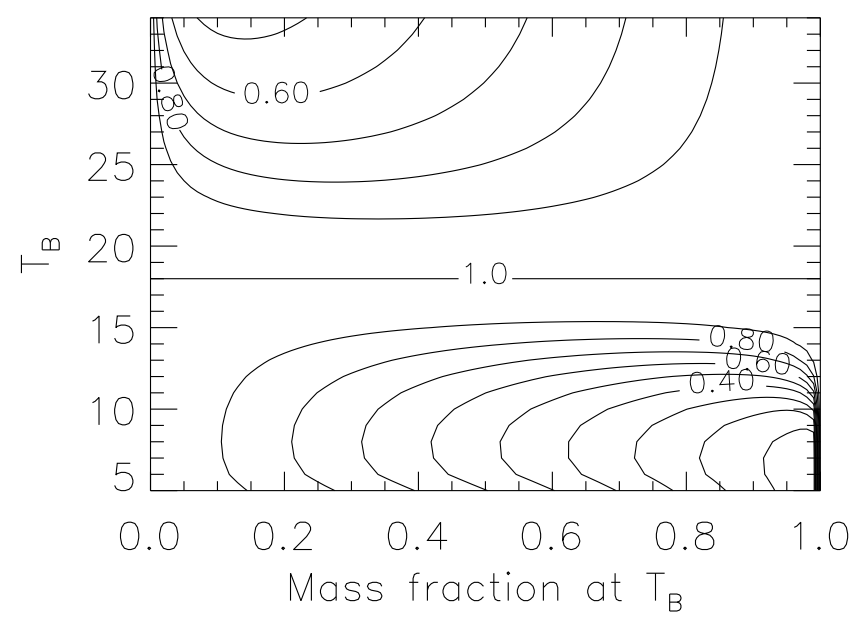

FIG. 2.-Ratio of recovered vs. true column density of dust using a single-temperature fit to two components. A fraction of dust $f_{B}$ at temperature $T_{B}$ is added to $18 \mathrm{~K}$ dust. The recovered column density is always lower than the true column density, with contours spaced in units of 0.1 .
VSGs are stochastically heated by the interstellar radiation field (ISRF) and cannot be characterized by a single temperature. VSGs undergo a sudden rise in temperature following absorption of a visible or UV photon, followed by a nearly continuous decline in temperature as the heat is radiated away in the form of many FIR photons. Such grains are assumed to be responsible for the stronger than expected Galactic cirrus emission observed by IRAS at $12-60 \mu \mathrm{m}$. The contribution from VSGs at the wavelengths that we use to fit the temperature $(\lambda>100 \mu \mathrm{m})$ is small (Draine \& Anderson 1985; Guhathakurta \& Draine 1989). Thus, the VSGs do not spoil our derivation of the column density due to classical grains. VSGs would be significant to our dust maps only if their density were much higher than expected and their distribution different from that of classical grains in the diffuse ISM.

\subsubsection{Recovering a Temperature Map}

The 100 and $240 \mu \mathrm{m}$ maps are too noisy to recover a reliable and independent dust color temperature at each DIRBE pixel. In the regions of low $\mathrm{S} / \mathrm{N}$ a filtering scheme is required. One option is to smooth the maps with a large beam, using the resulting maps to construct a heavily smoothed temperature map. This simple technique has the disadvantage of throwing away small-scale information in the compact dusty regions where the $\mathrm{S} / \mathrm{N}$ ratio is high. The spirit of most filtering methods is to produce a map with a fairly uniform $\mathrm{S} / \mathrm{N}$ ratio. An optimal scheme would smooth the data where there is no signal (and very little dust) and leave the data unsmoothed in the regions with large signal (and more dust). However, such schemes (e.g., the minimum-variance Wiener filter) have the disadvantage of overweighting low-S/N pixels near high-S/N pixels in such a way that bright sources have noisy halos.

One way to avoid artifacts such as halos around bright sources is to filter the maps on a local basis at each pixel. We assume that regions of very little dust have a uniform temperature. This allows us to combine the DIRBE maps with background averages taken at high Galactic latitudes, weighting in such a way that we preserve $S / N$.

To generate the temperature correction map from the zodiacal light-corrected DIRBE maps, we first remove 13 bright point sources from the DIRBE images (including M31, the LMC, and the SMC; see Table 1). We smooth both the 100 and $240 \mu \mathrm{m}$ maps with a Gaussian filter of FWHM $=1.1$, which determines the resolution of the resulting temperature map.

The dust effective color temperature is recovered from the ratio of intensities at 100 and $240 \mu \mathrm{m}$. Using the filtered DIRBE maps from equation (7), we define the ratio map

$$
\mathscr{R}=\frac{D_{100}^{S}}{D_{240}^{S}}
$$

where

$$
D_{b}^{S}=\mathscr{W} D_{b}^{Q}+(1-\mathscr{W}) \bar{D}_{b}^{Q} .
$$

Each filtered map $D_{b}^{S}$ is a weighted average of the local (Gaussian-smoothed) DIRBE flux and the background average flux determined at high Galactic latitude. The background average flux $\bar{D}_{b}^{Q}$ is determined separately in the north and south Galactic hemispheres. The weight function $\mathscr{W}$ determines how to filter the resulting $\mathscr{R}$ between the 
TABLE 4

\begin{tabular}{lrr}
\multicolumn{3}{c}{ CoEFFICIENTS FOR $K_{b}(\alpha, T)$} \\
\hline \hline Coefficient & $b=100 \mu \mathrm{m}$ & $b=240 \mu \mathrm{m}$ \\
\hline$a_{0} \ldots \ldots \ldots$ & 1.00000 & 1.00000 \\
$a_{1} \ldots \ldots \ldots$ & 2.18053 & -1.55737 \\
$a_{2} \ldots \ldots \ldots$ & -4.89849 & 0.74782 \\
$a_{3} \ldots \ldots \ldots$ & 2.38060 & \\
$b_{0} \ldots \ldots \ldots$ & -0.80409 & 0.89257 \\
$b_{1} \ldots \ldots \ldots$ & 3.95436 & -1.29864 \\
$b_{2} \ldots \ldots \ldots$ & -4.27972 & 0.61082 \\
$b_{3} \ldots \ldots \ldots$ & 1.70919 & \\
\hline
\end{tabular}

NoTE.-Coefficients for DIRBE color correction factors $K_{b}(\alpha, T)$, fitted using eq. (11). Fits assume an $\alpha=2$ emissivity model.

local quantity and the background average. Where $\mathscr{W}$ is small, the resulting $\mathscr{R}$ will have the high-latitude background value, as appropriate in regions of low $\mathrm{S} / \mathrm{N}$. In regions of high $\mathrm{S} / \mathrm{N}$ the weight function approaches unity, and $\mathscr{R}$ reduces to the local value of the flux density ratio. The advantages of this method are that it has no leakage from neighboring pixels and that the scale of the smoothing is constant. Therefore, noise generated by the ratio of two small uncertain numbers is suppressed. The background averages are assessed in each hemisphere by simply averaging the 100 and $240 \mu \mathrm{m}$ flux within the window $|b|>75^{\circ}$. The ratios are 0.661 in the north and 0.666 in the south.

We determine the weight function $\mathscr{W}$ by a minimumvariance analysis of the resulting ratio map $\mathscr{R}$. For this purpose we use a map of the standard deviation of measured $240 \mu \mathrm{m}$ flux at each point $\sigma_{240}$, reduced by the effective binning of the Gaussian smoothing of the maps. For the $240 \mu \mathrm{m}$ map the DIRBE errors are in the range $0.5<$ $\sigma_{240}<2.0 \mathrm{MJy} \mathrm{sr}^{-1}$, which is much larger than for the 100 $\mu \mathrm{m}$ map. Because the detector noise is negligible for the 100 $\mu \mathrm{m}$ data, we construct a weight function that minimizes the variance in the reciprocal of the color ratio $\delta^{2}(1 / \mathscr{R})$,

$$
\left\langle\delta^{2}\left(\frac{1}{\mathscr{R}}\right)\right\rangle=\frac{\sigma_{240}^{2} \mathscr{W}^{2}+\left(\bar{D}_{240}^{Q}-\bar{D}_{100}^{Q} / \mathscr{R}_{T}\right)^{2}(1-\mathscr{W})^{2}}{\left(D_{100}^{Q}-\bar{D}_{100}^{Q}\right)^{2} \mathscr{W}^{2}+\left(\bar{D}_{100}^{Q}\right)^{2}(1-\mathscr{W})^{2}},
$$

where $\mathscr{R}_{T}$ is an assumed mean color ratio. We use $\mathscr{R}_{T}=$ 0.55 , but the results are insensitive to this choice. Minimization of this function with respect to $\mathscr{W}$ leads to a quadratic equation whose solution is a map of the weight function

$$
\mathscr{W}=\mathscr{W}\left(D_{100}^{Q}, \mathscr{R}_{T}, \bar{D}^{Q}, \bar{D}_{100}^{Q}, \sigma_{240}\right) .
$$

The resulting $\mathscr{W}$-function is close to the simple expression $\mathscr{W}=D_{240}^{Q} /\left(D_{240}^{Q}+5\right)$ for typical noise levels in the DIRBE $240 \mu$ m map.

To convert from the ratio map $\mathscr{R}$ to effective color temperature, we need to know the emissivity model for the dust $\epsilon_{v}$ and the frequency response of the DIRBE instrument passbands $W(v)$. This information is combined to form a color correction factor $K_{b}$ for a passband $b$, using an $\epsilon_{v}=v^{\alpha}$ emissivity model,

$$
K_{b}(\alpha, T)=\int d v B_{v}(T) v^{\alpha} W(v) .
$$

Appendix B of the DIRBE Explanatory Supplement (1995) has tabulated $K_{b}(\alpha, T)$ for all their bands in the domain $0<\alpha \leq 2$ and $10 \leq T \leq 2 \times 10^{4} \mathrm{~K}$. These color correction factors are well fitted by a functional form

$$
K(\alpha, T)=\frac{\sum_{i} a_{i} \tau^{i}}{\sum_{j} b_{j} \tau^{j}}, \quad \tau \equiv \log _{10} T .
$$

Coefficients for these fits at $\alpha=2.0$ are presented in Table 4 . The fits are accurate to $1 \%$ at all temperatures. The measured flux in passband $b, D_{b}$, of dust emitting thermally with a $v^{\alpha}$ emissivity is

$$
D_{b}=K_{b}(\alpha, T) I_{b}(T),
$$

where $I_{b}$ is the actual intensity at frequency $b$.

Calculating the ratio $\mathscr{R}(\alpha, T)$ that DIRBE would measure for a given temperature and emissivity model, we have

$$
\mathscr{R}(\alpha, T)=\frac{D_{100}}{D_{240}}=\frac{K_{100}(\alpha, T) I_{100}(T)}{K_{240}(\alpha, T) I_{240}(T)} .
$$

We interpolate the function $K_{b}(\alpha, T)$ for 3000 temperatures and invert equation (13), yielding $T(R)$. The emissivity model $\alpha=1.5$ yields a color temperature $\sim 2 \mathrm{~K}$ hotter than $\alpha=2.0$, but the effect of this on the recovered dust column density is not significant, aside from an overall multiplicative normalization. Aside from this normalization, the final column density maps vary only at the $1 \%$ level for an $\alpha=1.5$ versus an $\alpha=2.0$ emissivity model. For all calculations that follow, we have chosen $\alpha=2.0$, in agreement with the Draine \& Lee (1984) dust model. The resulting temperature map is shown as Figure 3. The light regions on the temperature map denote warmer dust, while the darker regions denote cooler dust where the $100 \mu \mathrm{m}$ flux underestimates the column density of dust. Note that most of the sky is a neutral grey color, while several prominent highlatitude molecular cloud regions show up as dark filaments. A few isolated white (hot) spots correspond to the LMC, SMC, and O star regions near Ophiuchus and Orion.

We compute the column density of dust as the amount of emission we would expect in the DIRBE $100 \mu \mathrm{m}$ band if the dust were all at a reference temperature of $T_{0}=18.2 \mathrm{~K}$. The column density $D^{T}$ is expressed as the $100 \mu \mathrm{m}$ flux multiplied by a temperature correction factor $X$ :

$$
D^{T}=D_{100}^{Q} X
$$

where

$$
X(\alpha, T)=\frac{B\left(T_{0}\right) K_{100}\left(\alpha, T_{0}\right)}{B(T) K_{100}(\alpha, T)},
$$

and $B(T)$ is the Planck function. For an $\alpha=2.0$ emissivity model and an arbitrary reference temperature of $T_{0}=18.2$ $\mathrm{K}$, we combine equations (13) and (15) to solve for $X$ as a function of the DIRBE $100 \mu \mathrm{m} / 240 \mu \mathrm{m}$ ratio. The solution is well fitted by

$$
\begin{aligned}
\log _{10} X= & -0.28806-1.85050\left(\log _{10} \mathscr{R}\right) \\
& -0.02155\left(\log _{10} \mathscr{R}\right)^{2} .
\end{aligned}
$$

A temperature map $\mathscr{T}$ is recovered with the function,

$$
\begin{aligned}
\log _{10} \mathscr{T}= & 1.30274+0.26266\left(\log _{10} \mathscr{R}\right) \\
& +0.04935\left(\log _{10} \mathscr{R}\right)^{2} .
\end{aligned}
$$



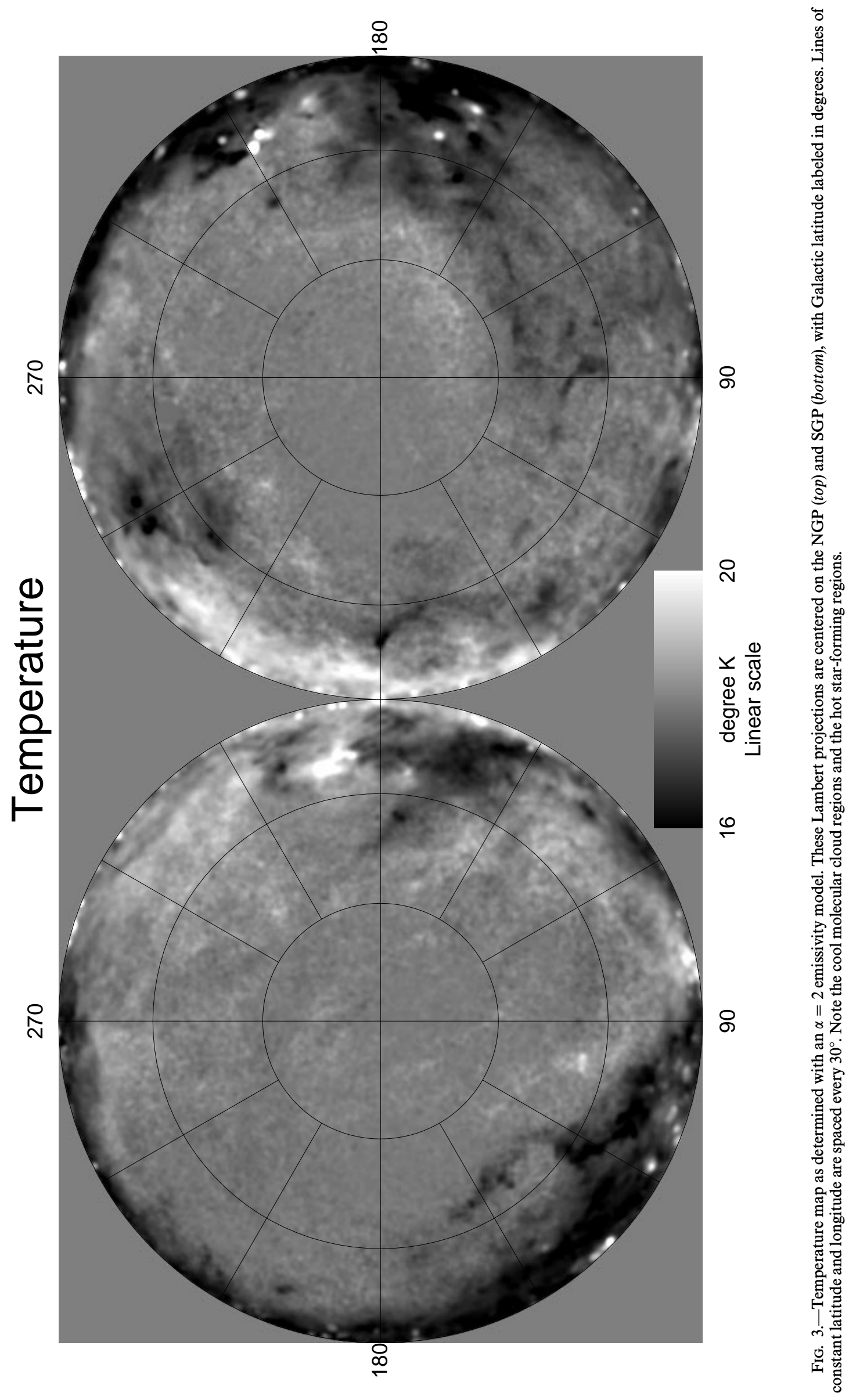
The maximum deviation of these fits is $0.8 \%$ in the domain $10<T<32 \mathrm{~K}$. The column density map $D^{T}$, recovered from equation (14), is our model for the Galactic dust at DIRBE resolution.

\section{FOLDING IN IRAS RESOLUTION}

The infrared maps from the $C O B E$ satellite have the advantages of being well calibrated and well corrected for zero-point drift. However, with a resolution of 0.7 FWHM, the $C O B E / D I R B E$ maps utterly fail to resolve important filamentary details in the cirrus and detect only the very brightest point sources. Therefore, we resort to the use of the older IRAS satellite data to improve upon this limited angular resolution.

The IRAS satellite observed $97 \%$ of the sky in four passbands, detecting some 250,000 point sources, including $\sim 20,000$ galaxies (Neugebauer et al. 1984). In addition to these point sources, the satellite discovered the infrared cirrus, especially visible in the $100 \mu \mathrm{m}$ band (Low et al. 1984). As the instrument was designed for point source detection (differential photometry), it is far from optimal for the absolute photometry required to make sense of the cirrus. In particular, the zero point from one scan to the next drifts considerably, leaving stripes with power on a range of scales, from a few arcminutes to several degrees.

The IRAS Sky Survey Atlas (ISSA; Wheelock et al. 1994) released by IPAC in 1991-1994 is the most useful presentation of the IRAS data for our purposes. The ISSA sky is divided into 430 plates $12.5 \times 12.5$ and spaced roughly every $10^{\circ}$ in right ascension and declination. The ISSA maps suffer from the zero-point drifts as well as residual zodiacal light. Despite the best efforts at IPAC to remove striping artifacts and zodiacal light, these features are still quite obviously present. This limits the usefulness of these maps for many applications.

We have reprocessed the ISSA data to create a $100 \mu \mathrm{m}$ map with the following properties:

1. ISSA plates are destriped, reducing the amplitude of the striping artifacts by a factor of $\sim 10$.

2. A deglitching algorithm was employed to remove small-scale artifacts that are not confirmed on other IRAS scans.

3. IRAS missing data areas were filled in with DIRBE data.

4. IRAS zero-point drifts were corrected to DIRBE on scales $\geq 1^{\circ}$.

5. Angular resolution is close to the IRAS limit with FWHM = 6. 1 .

6. Extragalactic objects were removed to a flux limit of $f_{100} \approx 1.2 \mathrm{Jy}$ at $|b|>5^{\circ}$.

7. Stars were removed to a flux limit of $f_{100} \approx 0.3 \mathrm{Jy}$ at $|b|>5^{\circ}$.

The combination of our destriping algorithm and the DIRBE zero point in effect removes zodiacal contamination from the ISSA maps. Section 3.1 discusses our Fourier destriping algorithm. Removal of deviant pixels, including asteroids, is described in $\S 3.2$. Section 3.3 describes the method used to combine DIRBE and IRAS data to form our final maps. The removal of 20,000 point sources is the topic of $\S 4$.

\subsection{Destriping the ISSA Maps}

IPAC took steps to destripe the ISSA plates, including a zodiacal light model and local destriping algorithm. However, serious striping remains. Fortunately, the scanning strategy of the IRAS satellite gives sufficient information to remove most of the striping artifacts that remain. Cao et al. (1996) have made important progress toward destriping the maps and deconvolving the IRAS beam reponse in small regions of the sky. However, due to the complexity of their algorithm and large amount of computing time required, it is impractical at this time to apply such a method to the full sky. We have developed an algorithm that is straightforward to implement and can easily be applied to the full sky.

We consider each ISSA plate separately and manipulate it in Fourier space. One ISSA plate and its Fourier transform are seen in Figures $4 a$ and $4 b$, where zero wavenumber is at the center. The rays emanating from the center are a clear signature of the striping in real space. The problem then becomes one of removing this excess power in Fourier space that is associated with the real-space stripes. Because the IRAS satellite scanned most of the sky 2 or 3 times, usually at a different angle, the contamination in Fourier space occurs at different wavenumbers for each scan. Thus, the contaminated wavenumbers from one scanning angle can be replaced with wavenumbers from the other scanning angles. The steps involved are (1) identifying the contaminated wavenumbers and (2) an optimal replacement strategy that allows for the lack of full coverage for many of the scans.

Because the stripes are radial in Fourier space, we parameterize the Fourier domain in polar coordinates $\left(k_{r}, k_{\theta}\right)$ for the purposes of this discussion, although it is discretely sampled in $k_{x}$ and $k_{y}$ for calculations.

For each ISSA plate there are 3 HCONs (hours-confirmed scans), referred to as $\mathrm{HCON}-1, \mathrm{HCON}-2$, and HCON-3. The composite map generated from these is called HCON-0, but when we refer to "the HCONs," we mean HCONs 1, 2, and 3. Many of these individual HCONs contain data with striping in only one direction. In general, however, a single HCON image may contain data taken in multiple scan directions and significant regions of missing data. In many cases $\mathrm{HCON}-3$ contains little or no data. For each HCON map there is a corresponding coverage map, which was used by IPAC to compute a weighted average of the three HCONs for each plate. (These coverage maps were kindly provided to us by S. Wheelock). The Explanatory Supplement (Wheelock 1994) also reports the use of a local destriper, but it appears to have had negligible effect in low-flux regions of the sky.

\subsubsection{Criterion for Bad Wavenumbers}

With each plate we begin by generating a composite map from the three HCONs, using the three coverage maps for weights. This is equivalent to the $\mathrm{HCON}-0$ composite maps released with the ISSA, except that no local destriper has been applied, and asteroids and other artifacts have not been removed.

Because our method is based on Fourier transforms where bright point sources may overwhelm many modes, we perform a crude point-source removal before any analysis. This consists of selecting objects or regions that are more than $0.7 \mathrm{MJy} \mathrm{sr}^{-1}$ above the median-filtered image. Several tests of this method indicate that it masks all significant point sources but not the striping. These sources are then replaced with the median of an annulus around 

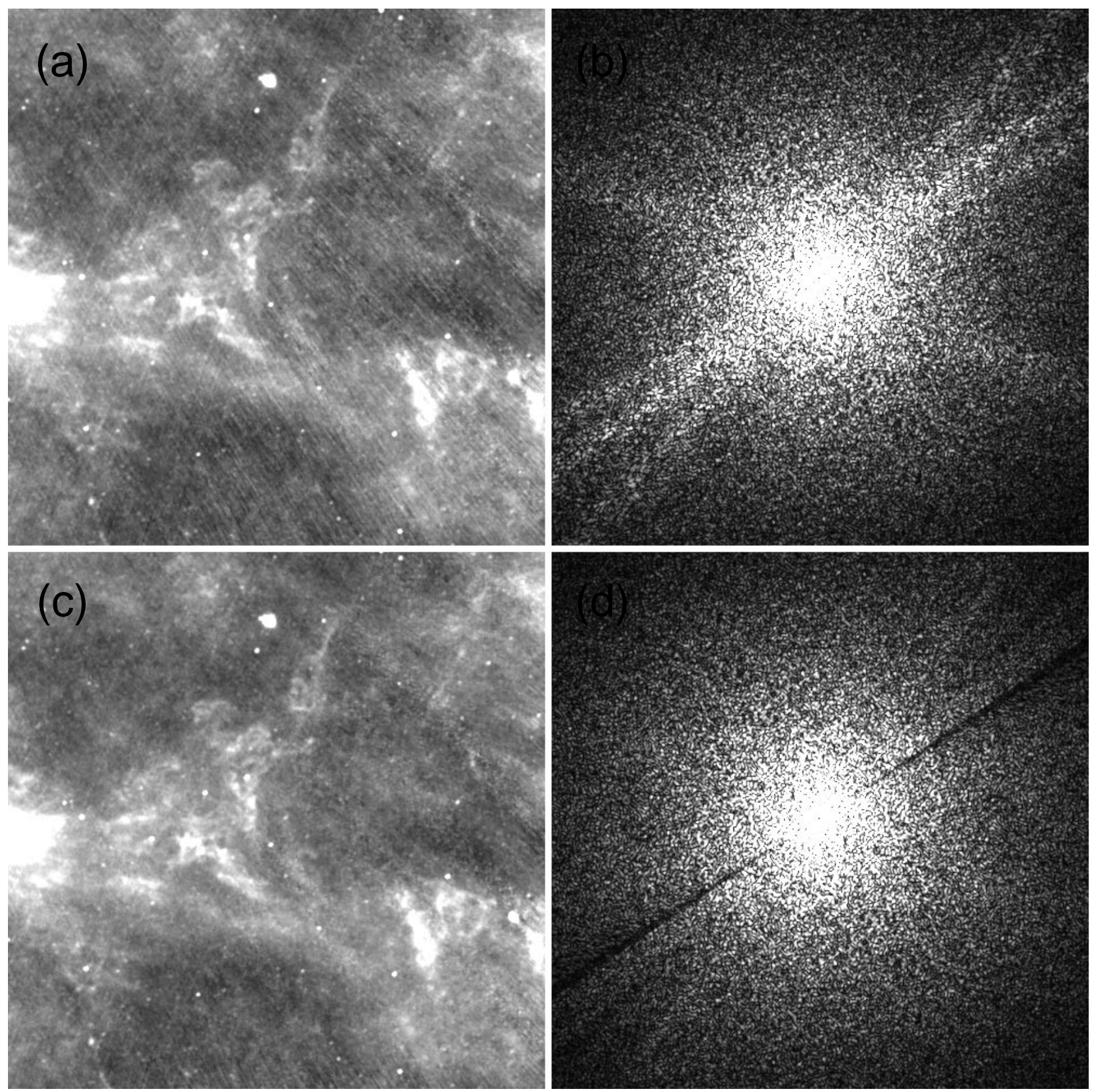

FIG. 4.-Fourier destriping of plate 379. (a) Raw ISSA HCON-0 image; $(b)$ FFT with wavenumber 0 in center; $(c)$ destriped image; $(d)$ FFT of destriped image. Note that one of the $k_{\theta}$ bins contains less power than the others. These are modes in which power from HCON-3 has replaced power in the other HCONs; however HCON-3 covers only half the plate, resulting in less power.

them. Because these sources are only removed when identifying contaminated modes, the details of this process are unimportant.

This composite $\mathrm{HCON}-0$ image (without point sources) is smoothed with a $6^{\prime}$ FWHM Gaussian to produce a baseline image that is used to fill in missing pixels in each of the three HCONs. This smoothing is enough to suppress most of the striping, and the replacement of missing pixels allows us to Fourier transform completely filled square images. Before transforming, we subtract the mean value from each image, apodize the edges, and embed the $500 \times 500$ image in a $1024 \times 1024$ array (which is equivalent to demanding isolated boundary conditions). The transform for a typical plate is shown in Figure $4 b$.

It is necessary to define a criterion with which to select the contaminated wavenumbers. We denote the fast Fourier transform (FFT) of HCON- $n$ as $F_{n}$ and a particular element of $k$-space as $F_{n}\left(k_{r}, k_{\theta}\right)$. We bin the Fourier domain into 90 azimuthal bins, each $2^{\circ}$ wide in $k_{\theta}$. A background power as a function of $k_{\theta}$ is determined by first finding the median at each $\theta$ and then median filtering the result. We define a power ratio $\gamma_{\theta}$ as the ratio of the power in each $k_{\theta}$ bin to the background power. By normalizing in this way, we expect $\gamma_{\theta}$ to be near unity, except in angular bins that are contami- 
nated by stripes. We somewhat arbitrarily choose $\gamma_{\theta}<1.2$ to be "good" power and $\gamma_{\theta}>1.6$ to be "bad." Between these two cuts, the information is deemed acceptable for retention, but poor enough to avoid replacing other "bad" power with it. In this way, "good" power is used where available, and we avoid corrupting real structures by not discarding power of ratio less than 1.6.

\subsubsection{The Destriping Algorithm}

Each HCON is destriped separately before they are combined to an averaged map. Because of the incomplete coverage in some HCONs, it would not be optimal to combine the images in Fourier space. Instead, for each HCON- $n$ we create a stripe image $S_{n}$, which is removed in real space. In this way we may utilize the good data in each HCON, even if only a fraction of the plate is covered.

A composite of "good" power, $F_{\text {good }}$, is generated by averaging all power at each $\left(k_{r}, k_{\theta}\right)$ with $\gamma_{\theta}<1.2$. Any mode with no "good" power is set to zero. (Modes with wavelengths larger than $1^{\circ}$ are not changed to avoid discreteness problems.) A bad pixel mask is produced for each HCON, indicating which regions of each $F_{n}$ are contaminated. The difference between $F_{n}$ and $F_{\text {good }}$ for bad modes only is inverse transformed to obtain $S_{n}$, the stripes corresponding to $\mathrm{HCON}-n$. This stripe map is subtracted from each raw HCON to produce individual destriped HCONs. Note that only stripes have been removed, and the destriped images retain all of the original point sources and other real structures. These destriped images are then deglitched (\$ 3.2) and averaged, weighted by the coverage maps, to obtain the final HCON-4 images.

Figure 5 shows the one-dimensional power spectrum of four plates before and after the destriping process. The destriping has reduced the high spatial-frequency variance by nearly a factor of 2 in some high Galactic latitude areas. In

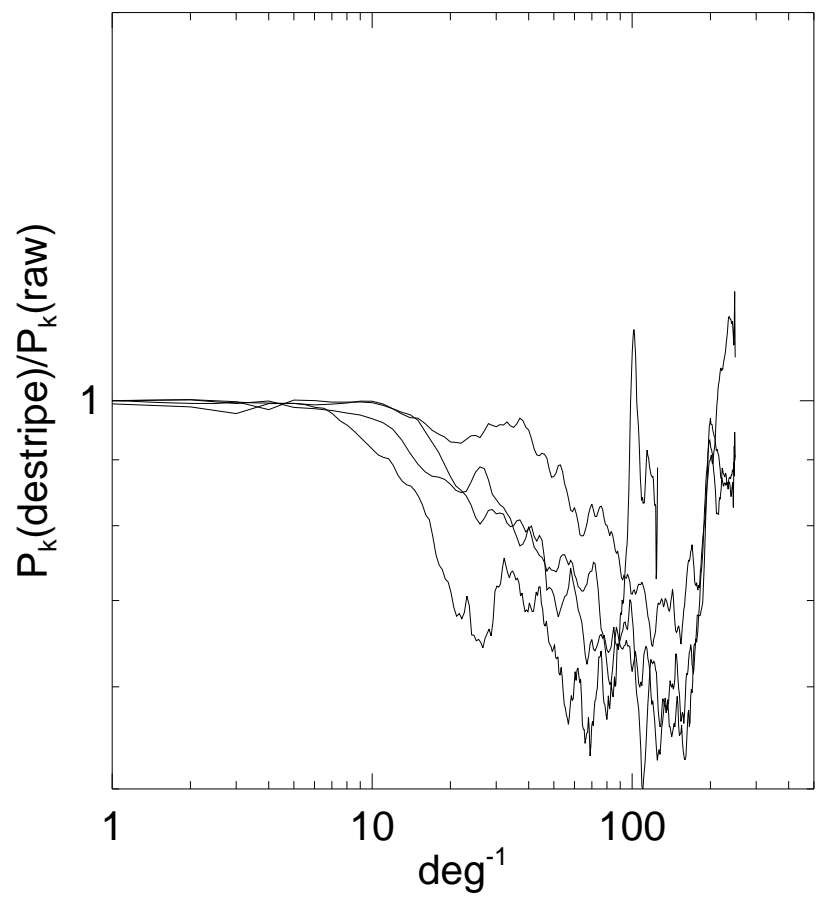

FIG. 5.-Ratio of power in destriped ISSA image vs. power in raw image. Ratios for four arbitrary plates are shown (plates 199, 216, 217, and 379). cases where the point-source subtraction fails, nothing is done to the plate. This occurs only at low Galactic latitude, where the striping artifacts are overwhelmed by cirrus emission. We have not found stripes to be important for the generation of a reddening map in these areas, so we have done nothing about them.

\subsection{Deglitching the ISSA Maps}

In addition to striping, the ISSA images contain other artifacts that must be removed. These anomalous features include transient sources, such as asteroids, and detector glitches that should have been removed from the timeordered data stream. Images with two or more HCONs allow identification of these artifacts by looking for discrepant pixels. We generically term this process "deglitching." IPAC chose to deglitch the ISSA images by visual inspection. This postproduction step removed anomalies from individual HCONs before combining them to produce HCON-0 images. Thus, HCON-0 differs from a coveragemap weighted average of the HCONs wherever anomalies were removed. Rather than rely upon visual inspection, we implement an automated deglitching algorithm.

The first task is to identify discrepant points. We compare each destriped HCON to a local median, filtered on a $10^{\prime}$ scale. Because some ISSA pixels have better coverage than others, they have lower noise. Therefore, we set the threshold for a deviant pixel at $2 / N^{1 / 2} \mathrm{MJy} \mathrm{sr}^{-1}$, where $N$ is the number of times IRAS observed that pixel. The pixels that deviate from the median-filtered background by more than this threshold are flagged, as are their immediate neighbors. Pixels flagged in all available HCONs are deemed to be "confirmed sources." These include real point sources, knots in the cirrus, and also pixels near regions of no data. In all these regions, asteroids cannot be differentiated from features that should remain in the map. However, pixels that are flagged in at least one, but not all, HCONs are flagged as "glitches." These pixels and their neighbors are replaced with data from the other HCONs. The reader should note that cirrus features are not modified by this algorithm.

Our deglitching algorithm affects less than $0.1 \%$ of the sky. For comparison, IPAC removes $0.47 \%$ of pixels at $|\beta|<20^{\circ}$ and $0.19 \%$ of pixels at $|\beta|>20^{\circ}$. The resulting destriped and deglitched ISSA maps are termed HCON-4.

Three planets were scanned by $I R A S$ and not adequately removed from the ISSA plates. These require special attention, as Saturn in particular is enormously bright compared to the background flux. Neptune and Uranus moved sufficiently between scans that they can be removed in one HCON while retaining coverage in the other HCONs. Because Neptune appears close to the Galactic plane, its surface brightness is comparable to the background level of $\sim 100 \mathrm{MJy} \mathrm{sr}^{-1}$. We remove it in each HCON with a circle of radius 7.5. Uranus is only incompletely removed from the ISSA plates, still causing hysteresis in the in-scan direction. We remove areas of $220^{\prime}$ in the in-scan direction and of $94^{\prime}$ in the cross-scan. Saturn is problematic, since it appears only in HCON-1 and HCON-2, and it moves very little between those scans. Because of this, we excise a circle of radius $2^{\circ}$ in all HCONs. This area, centered at $(l, b)=$ $\left(326^{\circ} 28,+51^{\circ} 66\right)$, is treated like the exclusion strip and filled with DIRBE data (see $\S 3.3$ ). The planet removal is implemented before the destriping algorithm and the deglitching procedure, afterward. 


\subsection{Combining ISSA with DIRBE}

The ISSA and DIRBE data are combined in such a way as to retain the small-scale information from IRAS and the large-scale calibration of DIRBE. The ISSA HCON-4 maps are processed to match the color response and zero point of the zodiacal-subtracted DIRBE $100 \mu \mathrm{m}$ maps on scales larger than $1^{\circ}$. Regions with no IRAS data are filled with DIRBE-resolution data. The maps are first projected into two $4096 \times 4096$ pixel polar projections, one for the north and another for the south.

The ISSA maps are first multiplied by a constant value $C$ to correct the IRAS gain approximately to that of DIRBE. The relative gain factors between DIRBE and IRAS are difficult to assess exactly, owing to the drifts in IRAS zero points. The DIRBE Explanatory Supplement presents a preliminary linear transformation between IRAS and DIRBE data based upon carefully selected regions (in an obsolete version of the supplement, but reprinted in Wheelock et al. 1994, Table IV.D.1). They find the IRAS brightness levels to be too high by $38 \%$ at $100 \mu \mathrm{m}$. We find the IRAS calibration to be too high by this value for the cirrus, yet it is consistent with DIRBE for bright point sources. We choose to recalibrate the $I R A S$ data globally with a compromise value of $C=0.87$. We also convolve the ISSA images with a FWHM $=3{ }^{\prime} .2$ Gaussian, $W^{\mathrm{G}}\left(3^{\prime} \cdot 2\right)$, bringing the effective $I R A S$ smoothing to 6.1 .

In order to correct the $I R A S$ zero point to that of DIRBE on scales greater than $1^{\circ}$, we construct a difference map between the two on these scales. This difference map $\mathscr{S}$ is added to the destriped IRAS map $I_{\text {des }}$ to yield a final map containing the small-scale information from IRAS with the large-scale calibration of DIRBE (after zodiacal correction):

$$
I_{\text {corr }}=C \cdot I_{\mathrm{des}} * W^{\mathrm{G}}\left(3^{\prime} \cdot 2\right)+\mathscr{S} .
$$

The difference map must be taken between the $I R A S$ and DIRBE data filtered to the same point-spread function. The procedure is complicated by the fact that neither the $I R A S$ nor DIRBE beams are Gaussian. The DIRBE point-spread function is approximately $42^{\prime} \times 42^{\prime}$ with power-law tails. Fortunately, for most of the sky, repeated scans from different directions result in an averaged DIRBE response approximated by a circular top hat. Therefore, the ISSA map is convolved with a circular top hat of radius $21^{\prime}$, $W^{\square}\left(21^{\prime}\right)$, while still in the $1^{\prime} .5$ pixel plates, and is then reprojected to a polar projection, introducing the same distortions that exist in the DIRBE projections. At this point, both maps are further smoothed by a FWHM = 40' Gaussian to obtain a final map with FWHM $=1.00$. The difference map is

$$
\mathscr{S}=\left[D^{Q}-C \cdot I_{\mathrm{des}} * W^{\mathrm{G}}\left(3{ }^{\prime} \cdot 2\right) * W^{\square}\left(21^{\prime}\right)\right] * W^{\mathrm{G}}\left(40^{\prime}\right),
$$

where $D^{Q}$ represents the $100 \mu \mathrm{m}$ DIRBE map after zodiacal correction (eq. [3]). The rms of the difference map is 0.9 $\mathrm{MJy} \mathrm{sr}^{-1}$ at $|b|>30^{\circ}$, which is representative of the zeropoint drifts in the IRAS/ISSA maps.

Due to the differing color responses, the residual of the smoothed ISSA and DIRBE maps has a few high and low regions near very bright sources. Other than NGC 253, these are all in the Galactic plane, the LMC, or the SMC. We mask these regions in the difference map to the median of an annulus around them (see Table 1). This treatment is not meant to be strictly correct and introduces substantial absolute errors relative to the rest of the map. However, on top of the sources the fractional errors are not significant, and this procedure eliminates halos and recovers the correct zero point nearby the sources.

Regions of missing ISSA data must be filled with DIRBE data in a way that treats the boundaries of the missing ISSA data properly. Both steps of the ISSA smoothing process are done in the following way: A mask, set to 1 for good pixels and 0 for missing data, is smoothed in the same way as the data. The missing data is zero filled so that it does not contribute to the smoothed result. After smoothing, mask pixels with values less than 0.5 are discarded, and the remaining pixels are properly weighted by dividing by the value in the smoothed mask. The mask generated by the second ISSA smoothing is applied to the DIRBE data set as well, so that in both maps pixels near a boundary only contain information from the "good" side of the boundary. Point sources just barely on the "bad" side should contribute equal tails to the "good" side, eliminating potential problems with the zero calibration.

The column density of dust that radiates at $100-240 \mu \mathrm{m}$ is recovered with

$$
D^{T}=I_{\text {corr }} X,
$$

where we have replaced the DIRBE $100 \mu \mathrm{m}$ flux with the corrected IRAS $100 \mu \mathrm{m}$ flux in equation (14). The temperature correction map $X$ is necessarily only at the DIRBE resolution of $\sim 1^{\circ}$, although the reprocessed $I R A S$ map has a final resolution of 6.1 .

\section{REMOVING POINT-SOURCE AND EXTRAGALACTIC OBJECTS}

For the purposes of an extinction map, only the Galactic infrared cirrus is of interest. Our dust maps are meant to trace the diffuse emission from dust as well as from localized clumps, such as Bok globules, which are typically on the scale of a few arcminutes. The stars, planetary nebulae, and other point (unresolved) sources in the ISSA must be eliminated. We also remove verified extragalactic objects down to a given flux limit. The resulting maps should be free of all contaminating sources and galaxies at $|b|>5^{\circ}$ and most such sources in select regions at lower latitudes.

\subsection{Strategy}

Our goal is to replace contaminating sources with the most likely value of the underlying $100 \mu \mathrm{m}$ emission. The most obvious solution is to subtract the flux multiplied by the PSF for each source. However, the shape of the PSF is neither Gaussian nor circularly symmetric, and its shape depends upon the IRAS scan directions at each position on the sky. Because of these uncertainties and because many extragalactic objects are not point sources anyway, we replace sources with a median value from the surrounding sky. For point sources, we replace pixels within a radius of either 5.25 (for $f_{100}<10 \mathrm{Jy}$ ) or 7.5 (for $f_{100} \geq 10 \mathrm{Jy}$ ). For extended sources, we replace pixels within the measured radius plus 1.5. The median is always taken from a surrounding annulus with the same area as its interior. In total, $1.2 \%$ of the ISSA pixels at $|b|>5^{\circ}$ are flagged as sources and replaced. The $\mathrm{LMC}, \mathrm{SMC}$, and $\mathrm{M} 31$, and sources in their vicinity are not removed. At latitudes $|b|<5^{\circ}$ we remove galaxies and stars only in those parts of the sky 
deemed unconfused by the PSCZ galaxy survey (W. Saunders 1997, private communication).

Sources are not identified from the IRAS $100 \mu \mathrm{m}$ maps because of the confusion with cirrus. Instead, sources are primarily identified from the $60 \mu \mathrm{m}$ band of the IRAS Point Source Catalog (PSC), where confusion with cirrus is less of a problem, and the spatial resolution is somewhat better.

\subsection{Extragalactic Sources}

Galaxies detected by the IRAS satellite have been extensively studied, and lists of such objects are readily available from the literature. Rice et al. (1988) have studied the largest galaxies detected by IRAS. Strauss et al. (1992) and Fisher et al. (1995) completed a redshift survey of IRAS galaxies to a flux limit of $1.2 \mathrm{Jy}$ at $60 \mu \mathrm{m}$ over most of the sky. The PSCZ redshift survey (W. Saunders 1997, private communication) covers a somewhat smaller area of sky to a lower flux limit of $0.6 \mathrm{Jy}$.

Many nearby galaxies are resolved in the ISSA maps and cannot be treated as point sources. Rice et al. have studied those galaxies with blue-light isophotal diameters greater than $8^{\prime}$ and report improved total flux densities from the $I R A S$ data. The 70 large galaxies with a total flux at $60 \mu \mathrm{m}$ exceeding $0.6 \mathrm{Jy}$ are removed from our maps as extended objects (excepting the LMC, SMC, M31, and M32, due to its proximity to M31). These objects are removed using a radius equal to $\frac{1}{2}$ their major axis, isophotal diameter at $B=25 \mathrm{mag} \operatorname{arcsec}^{-2}$, plus 1'.5 (as tabulated by de Vaucouleurs 1991). The radius of NGC 253 is increased to $36^{\prime}$ to remove IRAS hysteresis artifacts near this extremely bright source.

The IRAS 1.2 Jy Galaxy Survey identifies most infraredemitting galaxies at $|b|>5^{\circ}$ down to a flux limit of $1.2 \mathrm{Jy}$ at $60 \mu \mathrm{m}$ (Fisher et al. 1995). Fluxes for extended sources were systematically underestimated by the PSC and were improved with a process called ADDSCANing (Strauss, Davis, \& Huchra 1990). The galaxy survey employed a color selection, $f_{60}^{2}>f_{12} f_{25}$, which very efficiently discriminates between galaxies and stars. Each candidate source was classified based upon visual inspection of POSS and ESO plates, or upon inspection of CCD images if the plates were inconclusive. Planetary nebulae were identified spectroscopically. The survey identifies 5320 galaxies with $f_{60}>$ $1.2 \mathrm{Jy}$. In addition, 444 point sources made the flux and color cuts: 98 extragalactic $\mathrm{H}$ II regions, 210 stars, and 136 planetary nebulae. All these galaxies and point sources are removed from our maps, unless they coincide with the large (Rice et al.) galaxies already removed, or with the LMC, SMC, or M31. We ignore the sources classified as "cirrus or dark cloud," "unobserved," or "empty" fields, and two reflection nebulae.

The PSCZ redshift survey covers nearly the same region of sky as the $1.2 \mathrm{Jy}$ survey, but to a flux limit of $0.6 \mathrm{Jy}$ at 60 $\mu \mathrm{m}$. This survey employed an additional color cut, $f_{100} / f_{60}<4$, which retains approximately $97 \%$ of galaxies. A list of PSCZ identifications was provided by W. Saunders (1997, private communication) before publication. This list includes 15,285 galaxies, many of which are duplicates from the $1.2 \mathrm{Jy}$ survey. These galaxies, plus an additional 455 objects measured as unresolved by the ADDSCAN process, are removed from the dust maps.

The 1.2 Jy and PSCZ surveys did not have complete sky coverage. Two strips in ecliptic longitude (the exclusion strip) were not observed by the IRAS satellite, amounting to
$4 \%$ of the sky. Other regions, primarily at low Galactic latitude, are too confused by cirrus emission to allow reliable source identifications. These galaxy surveys divided the sky into approximately $1 \mathrm{deg}^{2}$ "lune" bins, from which masks were constructed. The $1.2 \mathrm{Jy}$ survey masks those bins at $|b|<5^{\circ}$, in the exclusion strip, or with more than 16 sources per deg ${ }^{2}$. These "confused" bins comprise $3.5 \%$ of the sky at $|b|>5^{\circ}$ and are primarily located in star-forming regions such as Orion-Taurus, Ophiuchus, and the Magellanic Clouds. The PSCZ survey masks a larger fraction of the sky at high latitudes $(8.5 \%)$, but includes $1.2 \%$ of the sky at low latitudes that is not confused.

We identify and remove a small number of galaxies by hand to make our dust maps as free as possible from contamination at $|b|>5^{\circ}$. The Point Source Catalog was searched for objects brighter than $1.2 \mathrm{Jy}$ that were masked from both the 1.2 Jy and PSCZ surveys. 195 satisfied the PSCZ color criteria for galaxies. Upon visual inspection of ISSA plates and the Digitized Sky Survey, 68 were identified as point sources or galaxies and removed from the dust maps.

The many faint galaxies that remain in our maps are not significant. As the median galaxy color is $f_{100} / f_{60}=2.0$, our adopted flux cut at $60 \mu \mathrm{m}$ roughly corresponds to $f_{100}>1.2$ Jy. Galaxies just below this limit will have a peak flux density of $\sim 0.12 \mathrm{MJy} \mathrm{sr}^{-1}$ at $100 \mu \mathrm{m}$, corresponding to $A(B) \approx 0.01 \mathrm{mag}$. Thus, the final extinction maps will have some small-scale contamination from extragalactic objects at that level. This contamination is very nearly uniformly distributed. In addition, very faint galaxies must contribute at some level to a uniform extragalactic flux. This contribution cannot be easily extrapolated from known galaxies, since the measured number counts $N \propto f_{60}^{-1.4}$ must flatten at low flux levels to prevent the total number and total flux from diverging. However, any uniform contamination is irrelevant, as it is degenerate with the constant term in the zodiacal contamination that has already been removed (§ 2.2.2).

\subsection{Stars}

We remove stars from the ISSA maps in all regions of $|b|>5^{\circ}$ and in select regions of lower latitude. Strauss et al. (1990) found that the infrared color criterion

$$
f_{60}^{2}<f_{12} f_{25}
$$

effectively discriminates stars from galaxies. We identify as stars all PSC objects that satisfy this color cut. At latitudes $|b|>5^{\circ}$ (and not near M31, LMC, and SMC) 4697 sources with $f_{60}>0.6 \mathrm{Jy}$ are selected for removal from the dust maps. At lower latitudes we only select stars in the unmasked regions of the PSCZ survey. A higher flux cut of $f_{60}>1.2$ Jy selects 97 stars in those low-latitude zones.

In Appendix A we show that any remaining fainter stars produce negligible contamination.

\section{USING THE MAPS TO MEASURE GALACTIC REDDENING}

For the past 17 years, almost all extragalactic observers have made reddening and extinction corrections using the maps of Burstein \& Heiles $(1978,1982)$. The BH maps derive the column density of dust from $\mathrm{H}$ I $21 \mathrm{~cm}$ flux. At northern declinations $\left(\delta>-23^{\circ}\right)$ the $\mathrm{BH}$ maps are modulated by local H I-to-dust ratios on scales of $13 \mathrm{deg}^{2}$, derived from the Shane-Wirtanen galaxy counts. The DIRBE/IRAS dust maps, on the other hand, directly 
measure the dust, and no corrections need be made for optically thick $\mathrm{H}$ I emission, for ionization, or for the formation of molecular hydrogen. Furthermore, the DIRBE/ IRAS maps are of uniform quality over the full sky, whereas the $\mathrm{BH}$ maps exclude all regions at $|b|<10^{\circ}(17.4 \%$ of the sky), as well as a region of $1080 \mathrm{deg}^{2}(2.6 \%$ of the sky) lacking $21 \mathrm{~cm}$ data. This latter region is near the south Galactic pole, approximately bounded by $-135^{\circ}<l<21^{\circ}$ and $b<-62^{\circ}$.

We limit the discussion of tests of our reddening map to two extragalactic reddening measurements. These tests provide confirmation that our dust maps are indeed suitable estimates of reddening, as good as or better than those of $\mathrm{BH}$. These tests also normalize the amplitude of reddening (in magnitudes) per unit of $100 \mu \mathrm{m}$ flux (in MJy $\mathrm{sr}^{-1}$ ). Our reddening estimates can be written simply as

$$
E(B-V)=p D^{T}=p I_{\text {corr }} X,
$$

where we seek the calibration coefficient $p . D^{T}$ represents the point source-subtracted IRAS-resolution $100 \mu \mathrm{m}$ map, corrected to a reference temperature of $18.2 \mathrm{~K}$ using the DIRBE temperature map (eq. [20]).

The reddening of external galaxies allows a straightforward calibration of our maps. For example, consider the sample of brightest cluster ellipticals of Postman \& Lauer (1995), for which $B-R$ colors are provided for 106 galaxies. These objects are at modest redshift $(z<0.05)$ and are well distributed over the sky. We use the $k$-corrected colors provided and apply our dust maps to estimate the reddening toward each galaxy, assuming $E(B-R)=$ $1.64 E(B-V)$ for normal grains with $R_{V}=3.1$ (see Appendix B). For a good normalization of the dust map, one expects no correlation between intrinsic $B-R$ for the galaxies and $E(B-V)$. Because the distribution of the intrinsic $B-R$ of the BCG galaxies is not Gaussian, it is best to use a nonparametric statistical procedure to test the correlation. We measured the Spearman rank correlation coefficient between extinction-corrected $B-R$ and $E(B-V)$. For calibration constants $p<0.0118$ or $p>0.0196$, the chances that a random distribution would have a correlation as large as observed is less than 5\%. Thus, our $95 \%$ confidence limits for the normalization of the maps is $p=0.016 \pm 0.004$. As confirmation of the procedure, we applied the same statistic to these galaxies using the $\mathrm{BH}$ reddening estimates:

$$
E(B-V)=q E^{\mathrm{BH}} .
$$

$E^{\mathrm{BH}}$ represents the $\mathrm{BH}$ reddening in $B-V$, limiting the analysis to the 99 galaxies with $\mathrm{BH}$ estimates. For a scaling factor in the range $0.66<q<1.05$, the $\mathrm{BH}$ reddening applied to the BCG galaxies leaves no significant correlation between intrinsic $B-R$ and foreground reddening, indicating that the $\mathrm{BH}$ normalization $(q=1)$ is acceptable.

A sharper test of the reddening calibration is made possible by using an auxiliary correlate to reduce the variance of the intrinsic color distribution. For example, the $\mathrm{Mg}_{2}$ index described by Faber et al. (1989) is expected to correlate closely with the intrinsic $B-V$ color for elliptical galaxies. For both increased metallicity and increased stellar population age, the line strength increases, and the colors become redder. Use of the $\mathbf{M g}_{2}$ index is ideal, since it is available for large samples and is not affected by reddening. We use the sample of 472 elliptical galaxies presented by Faber et al., which has very broad sky coverage, including many galaxies in rather dusty directions. Of this sample,
389 galaxies have photoelectric colors in $\leq 30^{\prime \prime}$ apertures, $\mathrm{Mg}_{2}$-index measurements, and estimates of $\mathrm{BH}$ reddening. Five of these galaxies are reported by D. Burstein (1997, private communication) to have suspect photometry and have been removed from our list (NGC 83, NGC 545, NGC 708, NGC 1603, and NGC 7617). The $B-V$ colors are listed in this catalog as being $k$-corrected and reddeningcorrected in a manner that deviates slightly from $\mathrm{BH}$ reddenings given in the published full-sky $\mathrm{BH}$ map. In one region of the sky $\left(230^{\circ}<l<310^{\circ},-20^{\circ}<b<+15^{\circ}\right)$ it was determined that the $\mathrm{BH}$ map is not reliable, and reddenings were assigned based upon deviations in the Mgcolor relation (Burstein et al. 1987). We have added the tabulated reddening corrections back to the colors to obtain the raw (only $k$-corrected) colors before proceeding with our analyses. We should note that the unreliable region lacks a dust-to-gas ratio estimate in the $\mathrm{BH}$ map construction.

Dave Burstein kindly sent us an updated version of the Faber et al. Table 1, in which the $\mathrm{Mg}_{2}$ estimates have been improved. For these objects we compute a linear regression of reddening-corrected $B-V$ against $\mathrm{Mg}_{2}$ with residuals $\delta(B-V)$. We compute the Spearman rank correlation coefficient of $\delta(B-V)$ versus the reddening estimate, again arguing that a good dust map will have no residual correlation. For the $\mathrm{BH}$ reddenings, as listed by Faber et al. (1989), or for the published BH reddening estimates, there is no significant residual correlation between $\delta(B-V)$ and the reddening estimate. Our formal normalization of the $\mathrm{BH}$ map is $q=0.90 \pm 0.09$, with the standard deviation in $\delta(B-V)$ reduced from 0.048 to $0.031 \mathrm{mag}$.

Applying the same procedure to the DIRBE/IRAS maps, we find a $95 \%$ confidence range $p=0.0184 \pm 0.0014$ for the calibration constant. For $p$ values in this range, the standard deviation of $\delta(B-V)$ is reduced to $0.028 \mathrm{mag}$. A plot of $B-V$ residuals from the $\mathrm{Mg}$-color relation versus reddening is shown in Figure 6, using both $\mathrm{BH}$ and DIRBE/ $I R A S$ reddening estimates. A different symbol type is used for galaxies in the north $\left(\delta>-23^{\circ}\right.$; squares $)$ versus the south $\left(\delta<-23^{\circ}\right.$; crosses $)$, as the $\mathrm{BH}$ maps do not utilize dust-to-gas ratio information for the southern points. Galaxies with no $\mathrm{BH}$ map values were not used in the fits, but are shown in the lower panel (asterisks). A slight trend in the
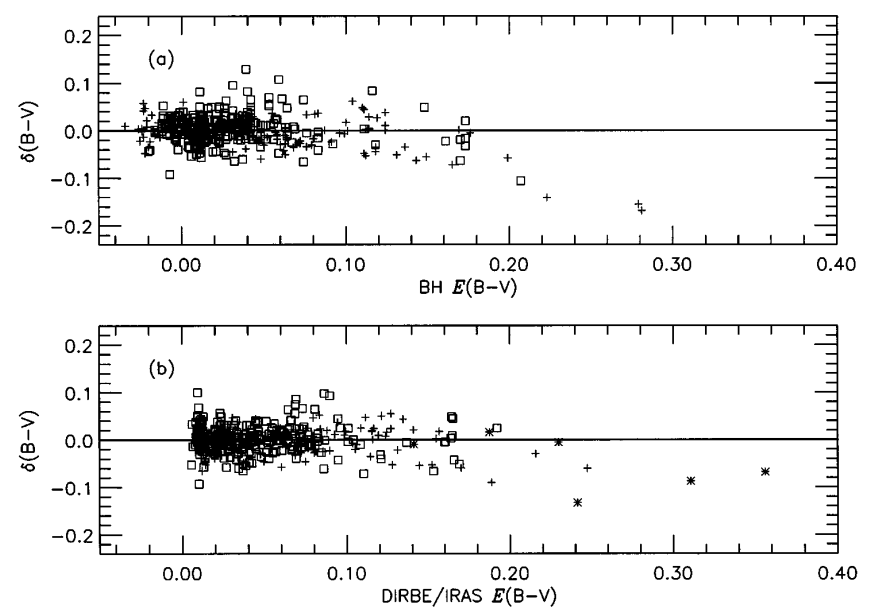

FIG. 6.-Residuals from the $B-V$ vs. $\mathrm{Mg}_{2}$ regression, plotted against foreground reddening, for $(a)$ the $\mathrm{BH}$ maps and $(b)$ the DIRBE/IRAS maps. Pluses represent galaxies at southern declinations where the $\mathrm{BH}$ maps lack dust-to-gas ratio information, and asterisks are those lacking any $\mathrm{BH}$ values. 
residuals is evident for both $\mathrm{BH}$ and DIRBE/IRAS corrections, in the sense that the highest reddening values appear to be overestimated. However, this trend is not statistically significant for the DIRBE/IRAS corrections. Note that the galaxies lacking BH estimates (asterisks) were not used in the fits, but still have reasonable color residuals using DIRBE/IRAS reddening corrections.

The larger size and reduced intrinsic color scatter of the Faber et al. sample leads to a much tighter constraint on $p$, one that is consistent with the value derived from the BCG sample. The $10 \%$ precision of the calibration is remarkably tight and is consistent with early estimates of IRAS reddening calibrations from Rowan-Robinson et al. (1991). (However, we note that IRAS-only estimates of the reddening severely suffer from the poorly constrained zero point, zodiacal contamination, and unaccounted variations in the dust temperature.)

The accuracy of the reddening maps can be estimated from the residuals in the $\mathrm{Mg}$-color relation. We assume that the errors in the reddening estimates $\sigma_{D-I}$ are fractional, increasing with increasing reddenings such that

$$
\sigma_{D-I}=f E(B-V) .
$$

We further assume that the intrinsic dispersion in the $\mathrm{Mg}$ color relation is $\sigma_{B V}=0.0257 \mathrm{mag}$ (including measurement errors), which is the measured dispersion for a subsample of galaxies in clean regions of the sky. A total error for the color of each galaxy is the quadrature sum of $\sigma_{D-I}$ and $\sigma_{B V}$. Using this model for the errors, one can determine the accuracy of the reddenings by increasing $f$ until the $\chi^{2}$ of the fit equals the number of degrees of freedom. For the $\mathrm{BH}$ reddening maps we find $f=0.29$, and for the DIRBE/IRAS reddening maps we find $f=0.16$. This demonstrates that the DIRBE/IRAS reddening estimates have an accuracy of $16 \%$, which is nearly a factor of 2 improvement over the BH estimates. In regions of low reddening, $E(B-V) \lesssim 0.1 \mathrm{mag}$, this data set indicates that DIRBE/IRAS and $\mathrm{BH}$ reddening estimates may be equally good.

We had originally attempted to use counts in cells of the APM galaxy survey (Maddox et al. 1990a, 1990b) as a normalization of the dust map. Extinction as measured by the DIRBE/IRAS column-density maps can be calibrated by studying the statistical covariance between the APM and DIRBE/IRAS dust maps: dusty regions have increased dust emission and diminished galaxy counts. However, we encountered a problem similar to that seen by Heiles (1976) when he attempted to compare reddening measures to the counts of Shane-Wirtanen galaxies. Using the APM counts we find a normalization $(p)$ that is approximately twice that described above. The extra sensitivity of the galaxy counts to dust is almost certainly a result of the catalog of galaxies being surface brightness limited as well as magnitude limited; increased foreground dust not only diminishes the total flux of the galaxy but diminishes the size of the isophotal aperture and increases the likelihood that the galaxy will be either classified as a star or not be counted at all. We shall provide details of this analysis in a separate paper (Finkbeiner et al. 1997).

\section{PROCESSING SUMMARY}

We have extensively processed the available far-IR data sets to generate a uniform-quality column density map of the dust that radiates from 100 to $240 \mu \mathrm{m}$. The major steps in the processing are summarized below:
1. The annual average DIRBE maps have been regenerated such that the same zodiacal dust column is observed at all wavelengths. Data with solar elongation $e<80^{\circ}$ have been deleted.

2. A zodiacal light model has been constructed from the DIRBE $25 \mu \mathrm{m}$ map. The model parameters were constrained by forcing the dust to trace the gas (as measured by the Leiden-Dwingeloo H I survey) at high Galactic latitudes.

3. Striping artifacts in the IRAS/ISSA $100 \mu \mathrm{m}$ maps were removed using a Fourier-space filtering algorithm.

4. Asteroids and non-Gaussian noise were removed from the IRAS/ISSA maps using a deglitching algorithm that compared multiple scans.

5. The IRAS and DIRBE $100 \mu \mathrm{m}$ maps were combined, preserving the DIRBE zero point and calibration.

6. Stars and galaxies were removed to a limiting flux of $f_{60}=0.6 \mathrm{Jy}$ over most of the sky at $|b|>5^{\circ}$ and over parts of the sky at lower latitudes.

7. The color temperature of the dust was derived from the DIRBE 100 and $240 \mu \mathrm{m}$ maps. A temperature correction map is used to convert the $100 \mu \mathrm{m}$ cirrus map to a map proportional to dust column.

8. The temperature-corrected dust map is calibrated to the reddening of external elliptical galaxies. The use of the $B-V$ versus $\mathrm{Mg}_{2}$ correlation allows us to set the calibration constant to $10 \%$ precision.

We have confirmation of the superiority of the new procedure by a test of the scatter of residual $B-V$ colors after regression against $\mathrm{Mg}_{2}$ line strength. The scatter of the regression is dominated by intrinsic scatter among the galaxies. We measure a reduction in the variance that is consistent with a fractional error in the reddening estimates of $16 \%$ for the new maps versus $29 \%$ for the $\mathrm{BH}$ estimates.

\section{DISCUSSION}

\subsection{The Nature of the Full-Sky Infrared Cirrus}

The new data show a wealth of filamentary structure that extends to the smallest scale resolved by our maps. A representative slice of the sky is shown in Figure 7, where we present the BH image, the Leiden-Dwingeloo $\mathrm{H}$ I image, the dust map with DIRBE resolution, and the final dust map with IRAS resolution. This slice displays part of the Polaris Flare on the left and the delicate structure of the highlatitude Draco molecular cloud complex (Herbstmeier, Heithausen, \& Mebold 1993; Mebold et al. 1985), also known as MBM complex 26 (Magnani, Blitz, \& Mundy 1985), to the right of center. The small-scale structure in the plot is at the resolution limits of the IRAS data. Higher resolution data would perhaps show even finer scale structure.

The full-sky dust map is shown as Figure 8 with a logarithmic stretch to emphasize the faint high-latitude features. The cirrus filaments arch across the Galactic poles, and between these filaments there are several low-density holes. The Lockman hole, the region of minimum flux in the $\mathrm{H}$ I maps $\left(l=150.5, b=53^{\circ}\right.$; Lockman et al. 1986), is one such low-density region, with a temperature-corrected flux of $0.39 \mathrm{MJy} \mathrm{sr}^{-1}$. The southern Galactic sky contains regions with even lower dust emission, perhaps by a factor of 2. The lowest column density holes are located at $l=$ $346.4, \quad b=-58.0$, and $l=239.7, \quad b=-48^{\circ} .6$, with temperature-corrected fluxes of $0.18 \mathrm{MJy} \mathrm{sr}^{-1}$. Unfor- 
(a)
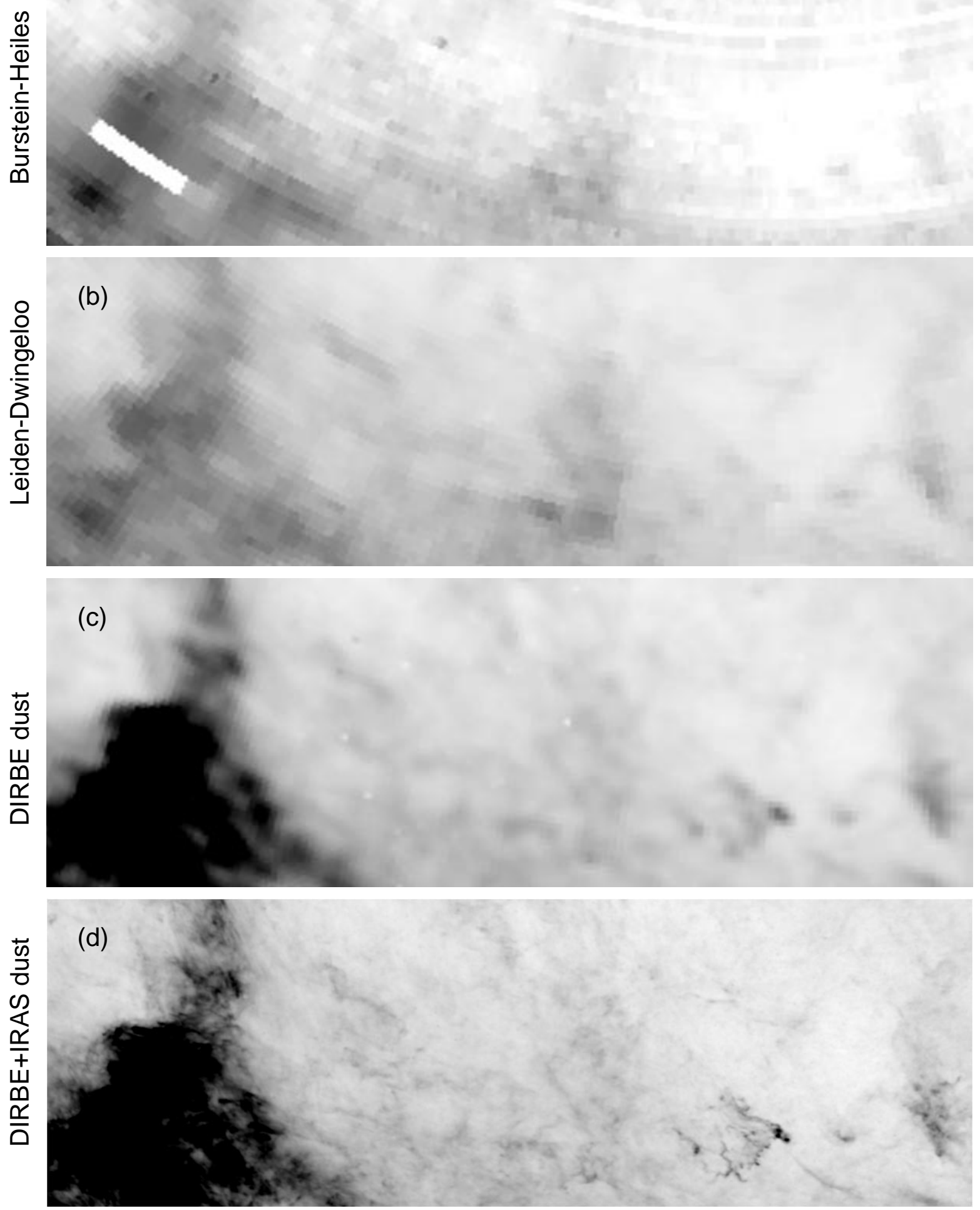

FIG. 7.-Slice of sky from (a) the BH map, (b) the Leiden-Dwingeloo H I map, (c) our dust map with DIRBE resolution, and (d) our dust map with IRAS resolution. The slice measures approximately $90^{\circ} \times 30^{\circ}$, centered at $l=100^{\circ}, b=+35^{\circ}$.

tunately, no high-quality $\mathrm{H}$ I data exists to verify that the lowest density holes are also minima in $\mathrm{H}$ I emission. A tabulation of dust properties for our low-density holes and the Galactic poles appears in Table 5. A large region of the southern Galactic sky $\left(b<-40^{\circ}, 160^{\circ}<l<320^{\circ}\right)$ appears to have very low dust emission and should therefore have the least foreground contamination for CMBR studies or large-scale structure analyses of redshift surveys. Projects in the northern hemisphere, such as the Sloan Digital Sky Survey and the north Galactic strip of the Two Degree Field (2dF) survey, may be more compromised by Galactic extinction. In particular, the north Galactic strip of the $2 \mathrm{dF}$ 


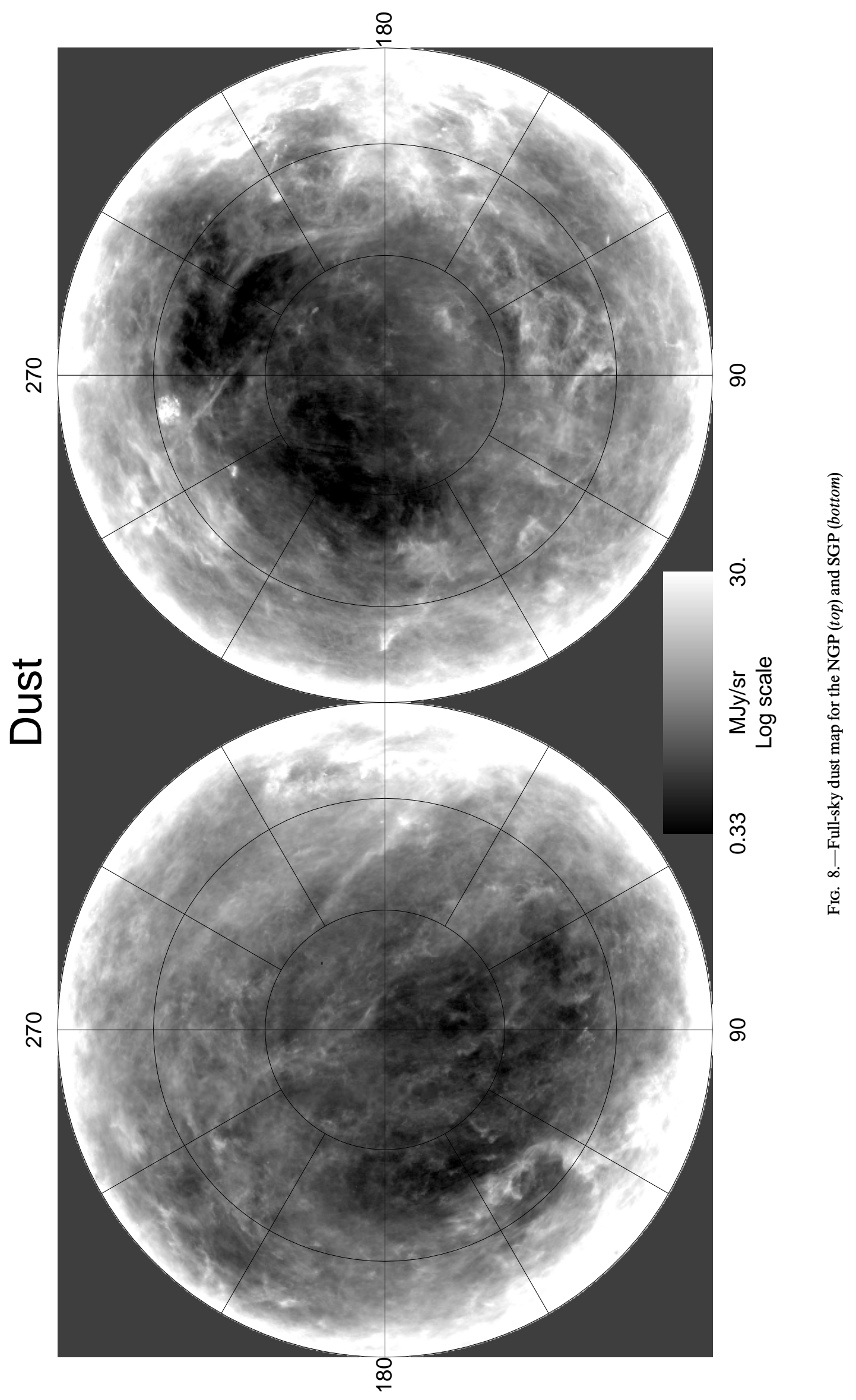


TABLE 5

Regions of Low Dust Column Density

\begin{tabular}{|c|c|c|c|c|c|c|c|}
\hline$\alpha_{200}$ & $\delta_{2000}$ & $\begin{array}{c}l \\
(\mathrm{deg})\end{array}$ & $\begin{array}{c}b \\
\text { (deg) }\end{array}$ & $\begin{array}{c}D^{T} \\
\left(\mathrm{MJy} \mathrm{sr}^{-1}\right)\end{array}$ & $\begin{array}{c}N(\mathrm{H} \mathrm{I}) \\
\left(10^{19} \mathrm{~cm}^{-2}\right)\end{array}$ & $D^{T} / N(\mathrm{H} \mathrm{I})$ & Comments \\
\hline $028 \ldots \ldots$ & -4244 & 318.4 & -73.7 & 0.35 & & & \\
\hline $051 \ldots \ldots$ & -2708 & 0.0 & -90.0 & 0.79 & 15.5 & 0.051 & SG pole \\
\hline $357 \ldots \ldots$ & -4850 & 257.1 & -48.4 & 0.33 & $\ldots$ & $\ldots$ & \\
\hline $359 \ldots \ldots$ & -4247 & 248.0 & -49.2 & 0.30 & $\ldots$ & $\ldots$ & \\
\hline $401 \ldots \ldots$ & -3425 & 235.2 & -49.1 & 0.20 & $\ldots$ & $\ldots$ & \\
\hline $403 \ldots \ldots$ & -3737 & 240.0 & -48.6 & 0.17 & $\ldots$ & $\ldots$ & \\
\hline $405 \ldots \ldots$ & -3550 & 237.4 & -48.2 & 0.20 & $\ldots$ & $\ldots$ & \\
\hline $444 \ldots \ldots$ & -5320 & 261.3 & -40.2 & 0.23 & $\ldots$ & $\ldots$ & \\
\hline $1036 \ldots \ldots$ & +5638 & 152.7 & 52.0 & 0.29 & 4.4 & 0.066 & \\
\hline $1048 \ldots \ldots$ & +5702 & 150.5 & 53.0 & 0.44 & 5.8 & 0.077 & Lockman hole \\
\hline $1251 \ldots \ldots$ & +2708 & 0.0 & 90.0 & 0.66 & 10.1 & 0.065 & NG pole \\
\hline $1335 \ldots \ldots$ & +3909 & 88.4 & 74.9 & 0.27 & 8.6 & 0.031 & \\
\hline $1342 \ldots \ldots$ & +4030 & 88.0 & 73.0 & 0.27 & 8.5 & 0.032 & \\
\hline $1344 \ldots \ldots$ & +5704 & 109.2 & 58.6 & 0.28 & 10.3 & 0.027 & \\
\hline $1354 \ldots \ldots$ & +4133 & 85.2 & 70.6 & 0.29 & 9.1 & 0.032 & \\
\hline $1410 \ldots \ldots$ & +3933 & 75.3 & 69.5 & 0.29 & 7.2 & 0.041 & \\
\hline $2243 \ldots \ldots$ & -4649 & 346.3 & -58.1 & 0.41 & $\ldots$ & $\ldots$ & \\
\hline $2322 \ldots \ldots$ & -4622 & 339.4 & -64.0 & 0.38 & $\ldots$ & $\ldots$ & \\
\hline
\end{tabular}

NotES.-Coordinates and values for the lowest column density regions in the dust map, the Lockman hole, and the Galactic poles. Units of right ascension are hours and minutes, and units of declination are degrees and arcminutes. Multiplication of $D^{T}$ by 0.0184 recovers the magnitudes of reddening in $E(B-V)$. H I column densities are for the full velocity range $\left(-450 \leq v_{\mathrm{LSR}} \leq+400 \mathrm{~km} \mathrm{~s}^{-1}\right)$ of the Leiden-Dwingeloo maps and are unavailable for the southern holes. All numbers are averages over a $1^{\circ}$ aperture.

is significantly more dusty than their southern region. However, the corrections derived from the new dust maps should prove adequate for most analyses.

In Figure 9 we show the power spectra of intensity fluctuations of the dust map calculated separately in eight independent patches of sky at high Galactic latitude. For each hemisphere we have extracted four quadrants in Galactic latitude bounded by $|b|>45^{\circ}$. The power spectra of these high-latitude patches have no preferred scale, but are reasonably well described as power laws with $P(k) \propto k^{-2.5}$. The amplitude of the power is different in each patch, which is indicative of extreme phase coherence in the cirrus structure of the Galaxy. To assume that the fluctuations can be approximated as random phase power is a completely inadequate description of this infrared cirrus and could lead to misleading estimates of Galactic foregrounds in CMBR experiments.

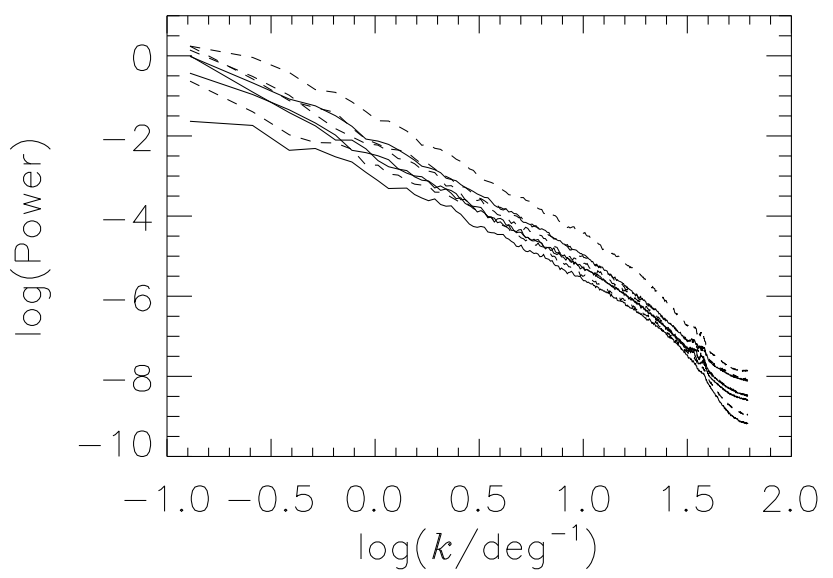

FIG. 9.-Power spectrum of dust. The four solid curves represent four quadrants in the north Galactic sky at $b>45^{\circ}$, while the four dashed curves represent four quadrants of the south Galactic sky.

\subsection{Differences between the Dust and $\mathrm{H}$ I Maps}

As seen in Figure 10, the correlation between the dust and the $\mathrm{H}$ I emission is remarkably tight for low flux levels, but has substantial scatter at higher flux levels. Because the $240 \mu \mathrm{m}$ map is on the Rayleigh-Jeans portion of the dust

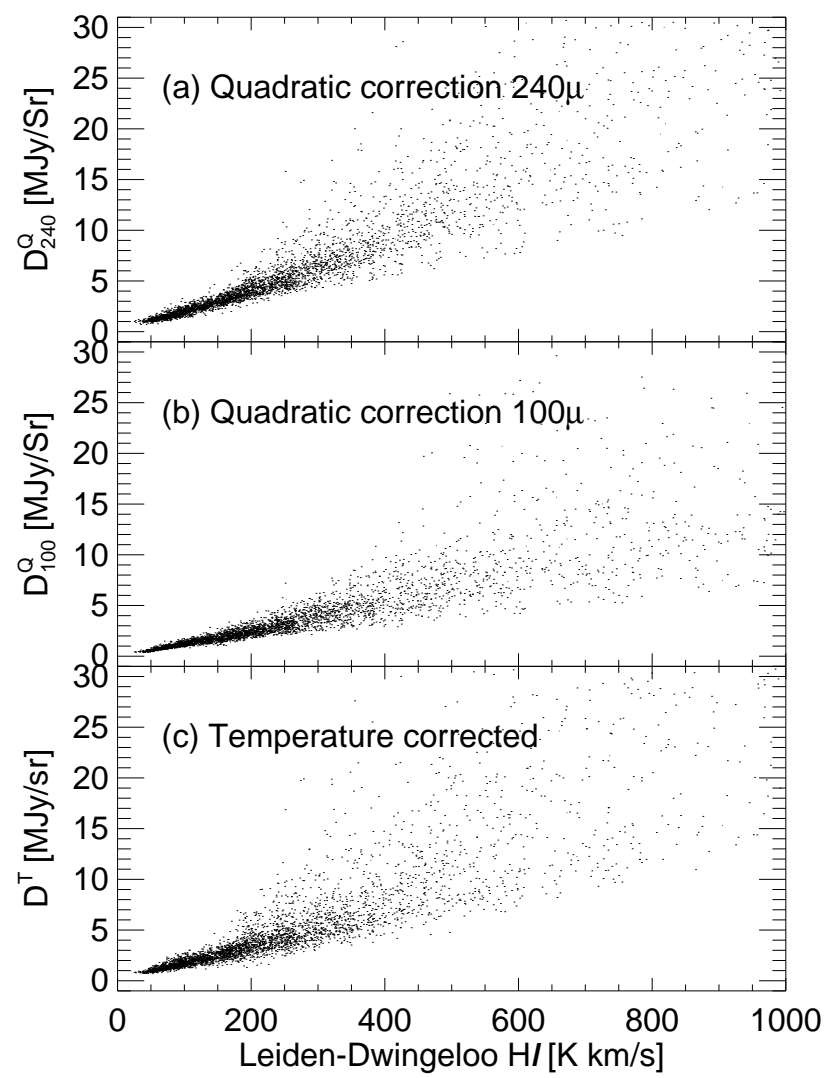

FIG. 10.-H I correlation with (a) DIRBE $240 \mu \mathrm{m}$ (corrected for zodiacal contamination), (b) DIRBE $100 \mu \mathrm{m}$ (also corrected), and (c) our derived dust column density. This plot demonstrates that the gas to dust relationship deteriorates at high flux levels. 
emission curve, one might expect it to correlate better with the $\mathrm{H}$ I than would the $100 \mu \mathrm{m}$ map. We see the opposite effect: there is more scatter in the $240 \mu \mathrm{m}-\mathrm{H}$ I correlation. We attribute this partly to the considerably larger noise of the $240 \mu \mathrm{m}$ map. But some of this increased scatter is undoubtedly generated by cool molecular cloud regions, in which much of the hydrogen is in molecular form, and the $100 \mu \mathrm{m}$ is exponentially reduced because of the lower temperature, while the $240 \mu \mathrm{m}$ emission is suppressed only linearly with temperature. Furthermore, the dust-H I scatter is nonnegligible even in the regions of low flux, where the gas is expected to be predominantly neutral. This scatter may indicate either fluctuations in the ionization fraction of the gas, extensive regions of $\mathrm{H}^{+}$, or variations in the dust-to-gas ratio within neutral zones.

A ratio map of temperature-corrected dust to the LeidenDwingeloo $\mathrm{H}$ I map is shown as Figure 11. The LeidenDwingeloo map includes $\mathrm{H}$ I gas from the full velocity range of the survey $\left(-450 \leq v_{\mathrm{LSR}} \leq+400 \mathrm{~km} \mathrm{~s}^{-1}\right)$ converted to dust column via the ratio $0.0122\left(\mathrm{MJy} \mathrm{sr}^{-1}\right) /(\mathrm{K} \mathrm{km}$ $\mathrm{s}^{-1}$ ) found in Table 2. Note that there are fluctuations in the ratio that are spatially coherent, indicating they are not instrumental noise but rather real features on the sky. Most of the high-latitude sky is a neutral grey, while regions of high dust-to-gas ratio appear as white. The most prominent of these features at high latitudes must be diffuse interstellar molecular clouds as well as regions of saturated $\mathrm{H}$ I emission, such as Orion $\left(l \approx 180^{\circ}, b \approx-20^{\circ}\right)$ and Ophiuchus $\left(l \approx 0^{\circ}, b \approx 15^{\circ}\right)$. The Polaris Flare $\left(l \approx 150^{\circ}, b \approx 40^{\circ}\right)$ is very conspicuous. Comparison of Figure 11 with Figure 3 shows that these regions of high dust-to-gas ratio often coincide with the regions of lower temperature, which is as expected, since the regions are optically thick in the UV and therefore shielded from the full ionizing flux of the Galaxy. Although searches for $\mathrm{CO}$ have failed to detect an abundance of molecular gas above the Galactic disk (e.g., Hartmann, Magnani, \& Thaddeus 1997), it is very likely that molecular hydrogen is very abundant in these cooler regions (Spaans \& Neufeld 1997).

Regions of low dust-to-gas ratio appear as black. These regions are primarily distant high-velocity $\mathrm{H}$ I clouds in which the dust, if present, is not as well illuminated by a UV radiation field, and is therefore too cold to emit at $100 \mu \mathrm{m}$. The Magellanic Stream is partially present in the south, while numerous high-velocity clouds are very prominent at high latitude in the north.

A general gradient of dust-to-gas ratio from Galactic center to anticenter is perhaps present in the northern sky, but if present in the southern sky, the gradient is obscured by the Orion Nebula. Even in the gray high-latitude regions, the dust-to-gas ratio map is not uniform and exhibits fluctuations of $\pm 15 \%$ amplitude that are coherent over scales of $10^{\circ}$. These modulations might indicate real gas to dust variation, or they may hint at some unresolved instrumental problems. For example, three parallel bands in the north are residuals of the imperfect zodiacal light removal, but their total modulation is only $15 \%$ peak to trough.

Figure 12 is a full-sky map of the DIRBE/IRAS reddening estimate minus the $\mathrm{BH}$ estimate for the region $|b|>10^{\circ}$. Recall that the BH maps are largely $\mathrm{H}$ I maps, with the zero point adjusted and with smooth variations in dust-to-gas ratio computed on the basis of galaxy counts. As expected, apart from an offset, the $\mathrm{BH}$ reddening maps are very close to the new reddening estimates over most high-latitude regions of the sky, rarely differing by more than 0.02 mag (aside from a global zero-point difference). But there are large systematic differences at low latitude and toward molecular clouds such as Orion and Ophiuchus. These modulations largely reflect the direct differences in the dust-to-gas ratio maps (Fig. 11). These coherent modifications of the reddening estimates are important for full-sky analyses of the galaxy distribution, particularly for studies of large-scale flow fields.

\subsection{The Reddening at the Galactic Poles}

The absolute zero point of dust column density in the Galaxy is both difficult to model from $21 \mathrm{~cm}$ or infrared emission and difficult to measure directly from stellar colors. The zero point of $21 \mathrm{~cm}$ maps are uncertain because of sidelobe contamination of the radio telescopes. Furthermore, the $21 \mathrm{~cm}$ maps completely miss any dust associated with ionized hydrogen. Although the DIRBE instrument chopped against a cold load to insure a stable zero point, the zero point of the combined DIRBE/IRAS dust maps is uncertain because of uncertain zodiacal contamination and a possible isotropic extragalactic background. Direct measure of the reddening is an equally difficult task. Extragalactic sources can not be used, because their intrinsic colors are not known. Studies of Galactic sources are limited to calibration of the dust column density between local and halo stars. Such comparisons may suffer from selection effects and do not sample Galactic dust beyond a few hundred parsecs.

The debate as to the reddening zero point has traditionally focused on the values at the Galactic poles. Although we find that the absolute minima in the dust column are not at the poles (see Table 5), most studies are near these two lines of sight. Some authors have claimed that the Galactic poles are essentially free of reddening, whereas others claim a reddening of order $E(B-V) \approx 0.02-0.05 \mathrm{mag}$, corresponding to an extinction $A(B) \approx 0.1-0.2 \mathrm{mag}$. This debate was reviewed by Burstein $\&$ Heiles in 1982 and has yet to be resolved.

Several large studies have been conducted to measure the colors of $\mathrm{A}$ and $\mathrm{F}$ stars near the Galactic poles. Color excesses from $u b v \beta y$ photometry for these stars with wellknown intrinsic colors are used to measure $E(b-y)$ reddening. Most of these studies argue for $E(B-V) \lesssim 0.01$ mag. The data of Hilditch, Hill, \& Barnes (1983) yield an average $E(B-V)=0.011 \mathrm{mag}$ for 34 stars at $b>75^{\circ}$ and more than $200 \mathrm{pc}$ above the plane. Furthermore, the subset of these stars in the quadrant $90<l<180^{\circ}$ show $E(B-V)=0.000$ mag. A similar northern hemisphere study by Perry \& Johnston (1982) is used to argue for negligible reddening for stars within $200 \mathrm{pc}$ distance toward the northern Galactic pole (NGP). In a companion study in the southern hemisphere, Perry \& Christodoulou (1996) find $E(B-V)=-0.010 \pm 0.016$ mag for 73 stars within $15^{\circ}$ of the southern Galactic pole (SGP).

A reanalysis of some of these data by Teerikorpi (1990) argues that these results are subject to statistical biases, suggesting that the average reddening reaches $E(B-V)=0.04 \mathrm{mag}$ at $400 \mathrm{pc}$ above the Galactic plane.

Our estimated reddening, averaged over $10^{\circ}$ in diameter, is $E(B-V)=0.015 \mathrm{mag}$ at the NGP and $0.018 \mathrm{mag}$ at the SGP. This corresponds to a blue-band extinction of $A(B)=0.065(0.080 \mathrm{mag})$ at the NGP (SGP), using an $R_{V}=$ 


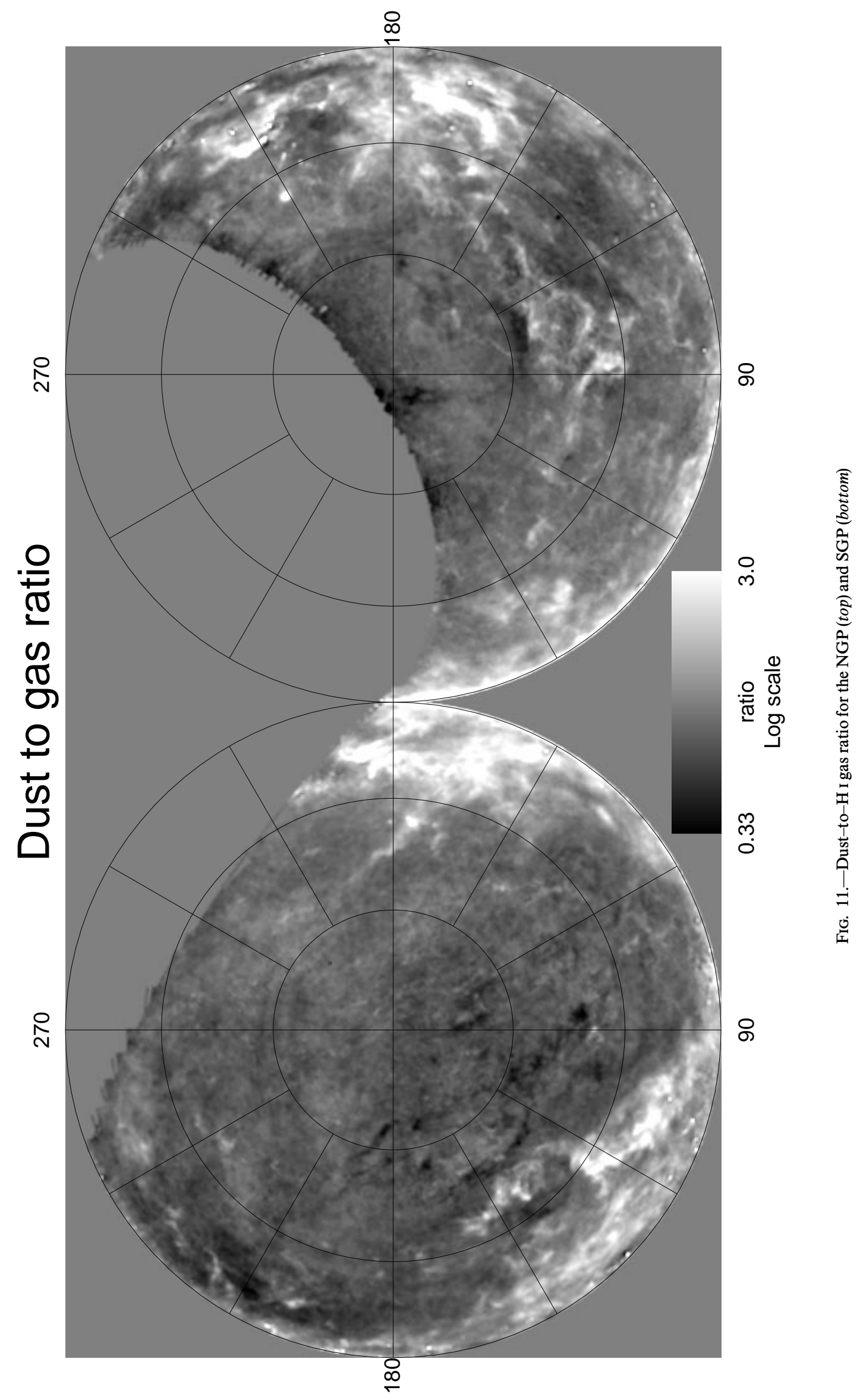




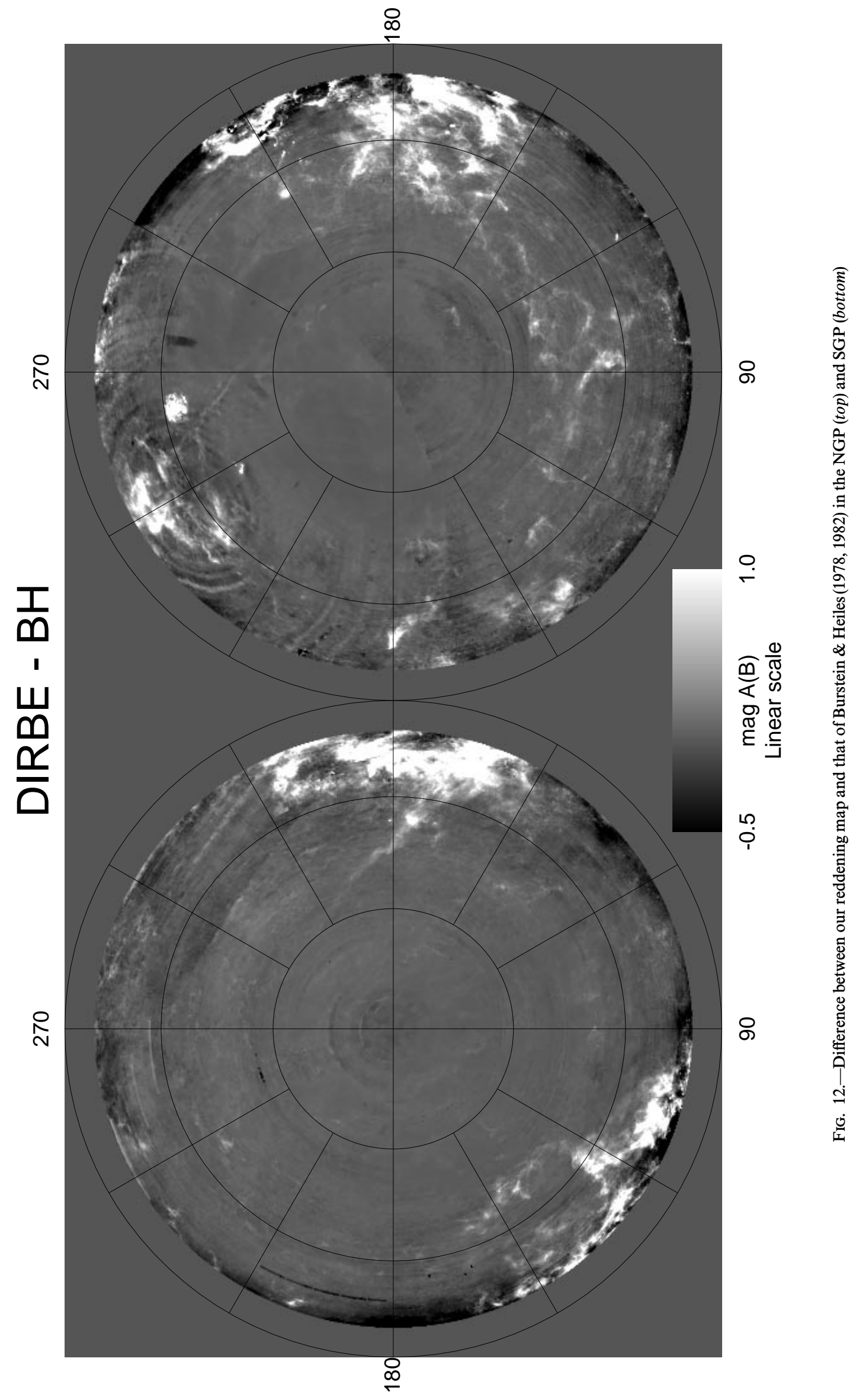


3.1 extinction law. We believe that a nonzero reddening at the poles is compelling for several reasons. First of all, we observe both $100 \mu \mathrm{m}$ and $\mathrm{H}$ I emission at the poles, not all of which could be scattered light or zodiacal emission, since it is structured and not entirely smooth at either pole. The fact that a linear dust-to-H I regression fits so well through all the low-flux regions suggests that this is truly material in the ISM. The gas has low velocity, so it is certainly part of the Milky Way, and so, therefore, must be the dust. If the A and $F$ star reddenings are not biased by selection effects, then this gas and dust must be more than several hundred parsecs above the Galactic plane.

It is theoretically unreasonable for the dust at high Galactic latitude to have fully evaporated. Although the dust at the poles might have been preferentially ablated by the shocks that formed the Local Bubble, such ablation would generally be expected to act oppositely to the coagulation that occurs in dense molecular regions. The grain size distribution should tip further toward smaller grains, thereby lowering the value of $R_{V}$ but not eliminating the small grains that dominate UV and optical extinction.

For many analyses a change in the zero point of dust reddening is irrelevant. But for studies such as photometry of distant supernovae, such an effect is quite important, and its neglect will lead to systematic errors in the inferred $q_{0}$. This arises because the extinction is not gray and more strongly affects the $B$ - or $V$-band spectra of nearby $\mathrm{SNe}$ than the $R$ - or $I$-band spectra of distant $\mathrm{SNe}$.

An offset is readily apparent between the DIRBE/IRAS reddening maps and those of Burstein \& Heiles. We measure a median difference of $0.020 \mathrm{mag}$ in $E(B-V)$ between the maps at high Galactic latitudes $\left(|b|>45^{\circ}\right)$, in the sense that the BH maps are systematically lower. For the reasons stated above, we believe the DIRBE/IRAS zero point to be more reliable. If one were to shift the DIRBE/ IRAS maps down by 0.020 mag, the lowest column density holes would have unphysical negative reddenings of $E(B-V)=-0.015 \mathrm{mag}$.

\subsection{Measurement of the CIB}

The search for the signature of an extragalactic background light (EBL) has a long history (Peebles 1971; Bond, Carr, \& Hogan 1986; Bond, Carr, \& Hogan 1991; Zepf \& Silk 1996; Guiderdoni et al. 1997; Franceschini et al. 1997). Such a background is inevitable and can be estimated based upon a star formation history and the corresponding injection of energy and metals into the interstellar medium at various epochs. The predicted EBL can be expressed as

$$
v I_{v}=0.007 \rho_{B} c^{2}(\Delta Z)(c / 4 \pi)\left(1+z_{f}\right)^{-1},
$$

where $\rho_{B}$ is the present baryon density and $\Delta Z$ is the overall metallicity produced at redshift $z_{f}$ (Bond et al. 1986). For $\Omega_{B} h^{2}=0.025$ and $\Delta Z=0.02$, this yields $v I_{v}=37 /\left(1+z_{f}\right)$ $\mathrm{nW} \mathrm{m} \mathrm{m}^{-2} \mathrm{sr}^{-1}$. A high background flux thus favors substantial metal production at low redshift. The emergent flux will appear either in the UV/visible/near-IR window if the star formation is not very dusty, or it will appear in the 100-300 $\mu \mathrm{m}$ window if the majority of the light in star forming regions is reprocessed by dust.

In the discussion of $\S 2.3$ above we described our regression of the dust maps on the $\mathrm{H}$ I data while removing the zodiacal foreground and a constant background term. Table 3 lists the derived background coefficients $B_{b}$ in $\mathrm{MJy}$ $\mathrm{sr}^{-1}$ as a function of the limiting ecliptic latitude cut $|\beta|$.
Note from this table that the inferred $B_{b}$ values drop as the fits are extended to lower values of $|\beta|$. This results from the inadequacy of the linear model for zodiacal foreground removal, especially for the $100 \mu \mathrm{m}$ data. But for fits with $|\beta|>20^{\circ}$, the 140 and $240 \mu \mathrm{m}$ fits stabilize and are significantly different from zero. The error bars are dominated by the large covariance between the measurement of $B_{b}$ and $A_{b}$, which becomes very extreme at large $|\beta|$ cuts. The error bars listed are the $95 \%$ confidence intervals, not including systematic effects. From variations of these results with ecliptic latitude cuts and from simulations with mock IPD maps, we estimate that systematic errors are comparable to the tabulated formal errors: $0.5,0.2$, and $0.2 \mathrm{MJy} \mathrm{sr}^{-1}$ at 100,140 , and $240 \mu \mathrm{m}$, respectively.

Based on these fits, we conclude that there does exist a well-quantified uniform background radiation at 140 and $240 \mu \mathrm{m}$. We prefer to use the coefficients derived from $|\beta|>30^{\circ}$ as the best compromise between an inadequate model at low $|\beta|$ and insufficient modeling leverage at high $|\beta|$. Our results are similar, although slightly higher, than those reported previously by Boulanger et al. (1996). The only significant difference between our procedure and that of Boulanger et al. is that we fitted all terms of the linear regression simultaneously. In perhaps more convenient units, a surface brightness of $1.0 \mathrm{MJy} \mathrm{sr}^{-1}$ translates to $v I_{v}=30,20$, and $12 \mathrm{nW} \mathrm{m}^{-2} \mathrm{sr}^{-1}$ at 100,140 , and $240 \mu \mathrm{m}$, respectively. The derived fluxes from Table 3 thus translate to an upper limit of $15 \mathrm{nW} \mathrm{m} \mathrm{m}^{-2} \mathrm{sr}^{-1}$ at $100 \mu \mathrm{m}$, and detected flux of $32 \pm 13 \mathrm{nW} \mathrm{m}{ }^{-2} \mathrm{sr}^{-1}$ at $140 \mu \mathrm{m}$, and $17 \pm 4 \mathrm{nW} \mathrm{m}^{-2} \mathrm{sr}^{-1}$ at $240 \mu \mathrm{m}$, where we have included the estimated systematic error.

We suspect that this isotropic flux is either extragalactic or is some sort of foreground directly related to the DIRBE telescope. No Galactic source is likely to be isotropic. We detect no hint of a dust layer distributed like $\csc |b|$ that is uncorrelated with the $\mathrm{H}$ I distribution, as might be expected from a uniform disk of ionized gas. Since these results derive from comparison to the $\mathrm{H}$ I distribution, they are sensitive to unknown offsets in the $\mathrm{H} \mathrm{I}$-dust relation. We have assumed that the Galactic $\mathrm{H}$ I-to-dust relationship has no zero offset, which leads us to more reddening than predicted by the $\mathrm{BH}$ maps. If we force our mean high-latitude reddening to match the $\mathrm{BH}$ maps, then the inferred CIB would increase.

A possible instrumental explanation for the strong signature at 140 and $240 \mu \mathrm{m}$ is given by Hauser (1996). Although the entire DIRBE telescope was operated at $2 \mathrm{~K}$, which should minimize radiation by the telescope mimicking an isotropic background, in these two channels there is a measurable radiative offset induced by junction field effect transistors (JFETs), operating at $70 \mathrm{~K}$, used to amplify the detector signals. Uncertainty in the correction for this effect is estimated to be 5 (2) nW m${ }^{-2} \mathrm{sr}^{-1}$ at 140 (240) $\mu \mathrm{m}$ (Fixsen et al. 1997), which is considerably less than the signal that we measure.

Presuming that this flux is indeed a cosmic infrared background, the inferred value is somewhat above that expected from the integrated star formation and dust reprocessing history of high-redshift galaxies, even if they are shrouded in dust (Guiderdoni et al. 1997). The measured background is 4 times larger than that expected based on an empirical summation of the observed luminosity density of galaxies (Malkan \& Stecker 1997). The numbers that we measure might be consistent with a strong burst of massive star 
formation in elliptical galaxies, if they are sufficiently dusty (Zepf \& Silk 1996). Puget et al. (1996) report an isotropic background detection from analysis of COBE/FIRAS data in the 400-1000 $\mu \mathrm{m}$ region, and our background measurements appear to be on the high side of the extrapolation of their measurements.

The EBL derived from the optical fluxes in the Hubble Deep Field (HDF), with modest extrapolation of the galaxy counts, is approximately $7.5 \mathrm{nW} \mathrm{m}{ }^{-2} \mathrm{sr}^{-1}$ (Madau 1997), a factor of $\sim 2$ smaller than that reported here. After masking detected sources in the HDF, Vogeley (1997) has shown that the remaining EBL, unless it is truly uniform, has a surface brightness that is at most a small fraction of the integrated light of the discrete sources. These results, together with recent observations of the UV continuum slope at high $z$ (Pettini et al. 1997), suggest that much early star formation was very dust enshrouded, reprocessing most of the UV, optical, and near-IR photons to the far-IR. An integrated far-IR background flux of such a magnitude, if correct, is a very promising sign for high angular resolution studies of the far-infrared. Space missions such as FIRST and the Infrared Space Observatory, and groundbased submillimeter observations with SCUBA on the JCMT and with the MMA will have a tremendous opportunity to resolve this background or to demonstrate that it does not actually exist.

A more detailed analysis of the CIB by the DIRBE team is currently underway (Hauser et al. 1998; Kelsall et al. 1998; Arendt et al. 1998; Fixsen et al. 1998). Instead of our simple empirical model of the zodiacal foreground, they have solved for a detailed three-dimensional model of the interplanetary dust. They provide a much more complete analysis of the separation of Galactic emission from the infrared background, and their results will undoubtedly supersede the preliminary measurements reported here.

\section{SUMMARY}

We have constructed a full-sky map of the Galactic dust based upon its far-infrared emission. The IRAS experiment provides high angular resolution at $100 \mu \mathrm{m}$, whereas the DIRBE experiment provides the absolute calibration necessary across several passbands to map the dust color temperature and to convert $100 \mu \mathrm{m}$ emission to dust column density. Point sources and extragalactic sources have been removed, leaving a map of the infrared cirrus. This dust map is normalized to $E(B-V)$ reddening using the colors of background galaxies. The final maps have a resolution of 6.1 and are shown to predict reddening with an accuracy of $16 \%$.

The new dust map leads to reddening estimates quite consistent with the Burstein-Heiles maps in most regions of the sky, with the new maps proving to be twice as reliable. These new maps are certainly to be preferred in regions of high extinction, where $\mathrm{H}$ I-based maps suffer from saturation of the $21 \mathrm{~cm}$ line or insensitivity to molecular hydrogen. Further tests are encouraged to determine the accuracy of our predicted reddenings and extinction. In particular, the accuracy of the maps at $|b|<10^{\circ}$ has yet to be established.

The maps will undoubtedly prove useful for analyses of current and future CMBR experiments, as well as a host of
Galactic structure studies. For example, it will be of interest to determine whether the temperature variations observed at high latitude are consistent with molecular line observations, and whether better constraints on the distance to the high-latitude molecular clouds can be obtained. Molecular line observations of several of the regions of low dust-to$\mathrm{H}$ I ratio (i.e., the high-velocity clouds) will give information on the metal abundance in these regions, which might lead to better constraints on their distance from the plane of the Milky Way.

We show that the dust correlates very well with the available $\mathrm{H}$ I maps over most of the sky. The ratio of dust to gas can be used to flag molecular clouds on one extreme and high-velocity $\mathrm{H}$ I clouds on the other. We note that the lowest regions of dust emission occur in the southern Galactic sky, in a region where there exist no high-quality $\mathrm{H}$ I maps. The south Galactic sky has less power in the dust and should be preferred for CMBR experiments and largescale Galaxy surveys.

We argue that Burstein \& Heiles (1982) underestimate reddening by $0.020 \mathrm{mag}$ in $E(B-V)$. Our predicted extinction at the Galactic poles is $A(B)=0.065 \mathrm{mag}$ (north) and $A(B)=0.080 \mathrm{mag}$ (south) on $10^{\circ}$ scales. There do exist holes that have extinction as low as $A(B)=0.02 \mathrm{mag}$; the most prominent of these are listed in Table 5 . We find that the lowest column density dust holes are in the southern Galactic sky, but it is not known if these are also the regions of lowest $\mathrm{H}$ I. If these selected regions are indeed low in $\mathrm{H} \mathrm{I}$, then they should become preferred directions for deep imaging and spectroscopy of extragalactic sources in the soft X-ray bands, where Galactic extinction is a severe problem.

In the process of generating these maps and removing the zodiacal foreground, we have detected what appears to be a significant far-IR background flux, approximately 32 and $17 \mathrm{nW} \mathrm{m}^{-2} \mathrm{sr}^{-1}$ in the 140 and $240 \mu \mathrm{m}$ bands, respectively. This flux is surprisingly high, higher than the integrated light seen in the Hubble Deep Field. If our measurement is a detection of the CIB and not some artifact associated with the DIRBE instrument, this suggests that early star formation was heavily dust enshrouded in most of the universe.

The dust maps are publicly available, as described in Appendix C.

The COBE data sets were developed by the NASA Goddard Space Flight Center under the guidance of the COBE Science Working Group and were provided by the NSSDC. We thank Sherry Wheelock of IPAC for providing us with ISSA coverage maps. We thank Steve Maddox for supplying the APM maps, Will Saunders and the PSCZ team for providing positions and identifications from the PSCZ survey, and Tod Lauer for providing the BCG data. We are especially indebted to Carl Heiles for stimulating discussions and invaluable guidance during the course of this project. Further discussions with Dave Burstein and Chris McKee were extremely useful. All the analysis described in this paper was performed in IDL, which increased our efficiency by an enormous factor. DPF was partially supported by an NSF Graduate Fellowship. This work was supported in part by NASA grant NAG 5-1360. 


\section{APPENDIX A}

\section{REMAINING STELLAR CONTAMINATION}

Stars have been removed from our map to a flux level of $f_{100} \approx 0.3 \mathrm{Jy}(\S 4.3)$. At $100 \mu \mathrm{m}$ these sources contribute a total of $26,700 \mathrm{Jy}$ at $|b|>5^{\circ}$, amounting to a mean flux density of $0.0026 \mathrm{MJy} \mathrm{sr}^{-1}$. With a median star color of $f_{100} / f_{60}=0.4$, our adopted flux cut at $60 \mu \mathrm{m}$ roughly corresponds to $f_{100}>0.3 \mathrm{Jy}$. Stars just below this limit have a peak flux density of $\sim 0.03$ MJy sr ${ }^{-1}$ at $100 \mu \mathrm{m}$, corresponding to $A(B) \approx 0.003$ mag. Thus, the contamination from remaining individual stars is very small in the final extinction maps.

The concern about remaining contamination from stars is that, although small on average, it may increase dramatically at low Galactic latitudes, where the star density is very high. We show that this is not a problem by extrapolating to the contribution from stars below our flux limit. In order to trace the star counts to low flux and low Galactic latitude, we restrict this analysis to a strict color cut that limits cirrus confusion: $0.1<f_{60} / f_{25}<0.3 ; 0.03<f_{60} / f_{12}<0.15 ; f_{100} / f_{60}<2$.

This strict color cut retains $70 \%$ of the stars, while efficiently excluding the cirrus. The color-color plane in Figure 13 compares it to the looser cut, $f_{60}^{2}<f_{12} f_{25}$. The distribution of stars (satisfying this strict color cut) as a function of flux is fitted by

$$
\Delta \log N=2.37-0.84\left(\Delta \log f_{60} / 0.1\right) .
$$

With a slope in the range $-1<m<0$, the number of stars at faint flux levels diverges, but the flux from those sources converges. This allows us to normalize the flux from stars fainter than a given flux cut $f_{\text {cut }}$, relative to the number of stars brighter than that cut:

$$
\frac{\text { Flux of stars }<f_{\text {cut }}}{\text { Number of stars }>f_{\text {cut }}}=\left(\frac{-m}{1+m}\right) \frac{f_{\text {cut }}}{\mathrm{Jy}} \text {. }
$$

For a flux cut $f_{\text {cut }}=0.6 \mathrm{Jy}$, this ratio is $3.2 \mathrm{Jy}^{-1}$. As the number of stars must converge, this ratio represents an upper limit to the flux from faint stars. Next, we trace the distribution of stars as a function of Galactic latitude. Again to minimize cirrus confusion, we limit ourselves to the strict color cut and brighter stars $\left(f_{60}>1.2 \mathrm{Jy}\right)$. The distribution of stars roughly corresponds to a csc $|b|$ law for $|b|>10^{\circ}$. At lower latitudes, the PSC is losing stars primarily because of confusion with cirrus. At $|b|=10^{\circ}$ the cirrus has a surface density that is typically 17 times brighter than a $1.2 \mathrm{Jy}$ point source in the ISSA $60 \mu \mathrm{m}$ maps.

We combine the flux and latitude distribution of stars to estimate the contamination from faint stars. Using a mean color for stars of $f_{100} / f_{60}=0.54$, we plot the estimated contribution from stars fainter than $f_{60}=0.6 \mathrm{Jy}$ in the $100 \mu \mathrm{m} \mathrm{maps} \mathrm{(Fig.} \mathrm{14).}$ The contamination is approximated by the functional form

$$
F_{100}=2 \times 10^{-4} \csc |b| \mathrm{MJy} \mathrm{sr}^{-1} .
$$

For comparison, we overplot the total $100 \mu \mathrm{m}$ flux from the PSC stars explicitly removed from the maps. This demonstrates that the faint stars are expected to contaminate the $100 \mu \mathrm{m}$ maps at a level less than $0.01 \mathrm{MJy} \mathrm{sr}^{-1}$ for $|b|>5^{\circ}$. This is not significant, as it represents contamination in derived $A(B)$ values of less than $10^{-3} \mathrm{mag}$.

\section{APPENDIX B}

\section{EXTINCTION IN DIFFERENT BANDPASSES}

We have shown that the DIRBE/IRAS dust maps faithfully trace the dust responsible for reddening of blue light (§ 5). We need some method of extrapolating the reddening in $B-V$ to reddening and extinction in other passbands. Measuring such relative extinctions is a well-developed industry, albeit one still fraught with some controversy. In particular, the composition of interstellar dust is not well known, and nor is its variation within the Galaxy.

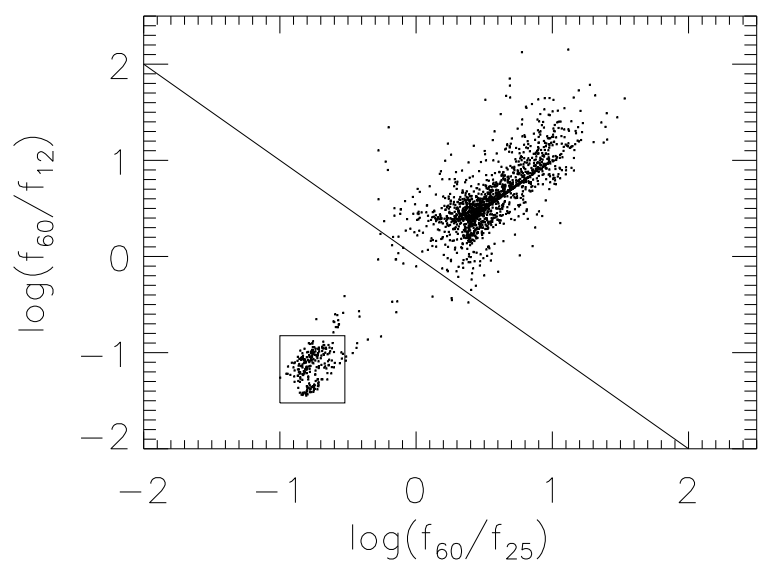

Fig. 13.-Color-color diagram for PSC sources. The diagonal line efficiently discriminates between galaxies (above the line) and stars (below the line). The square box is a strict color cut that retains $70 \%$ of stars. For clarity, only $1 / 10$ of the stars are plotted. 


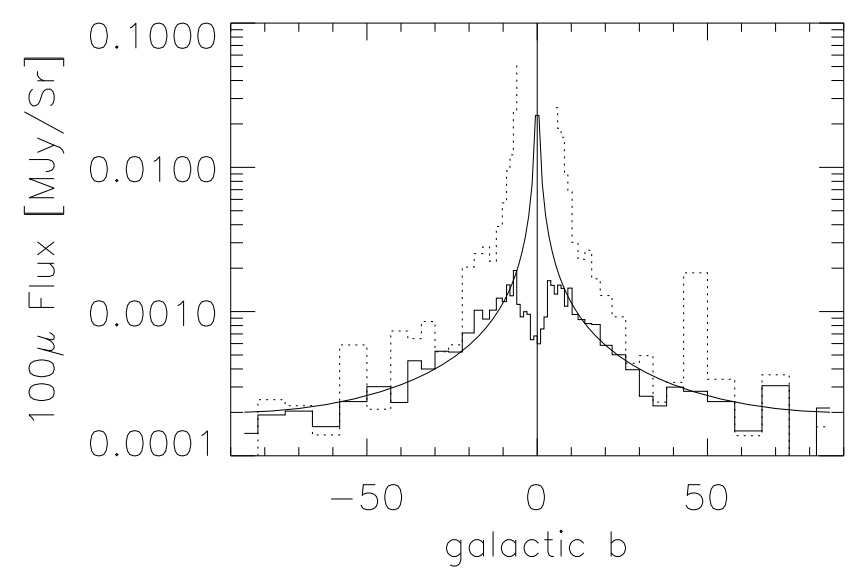

FIG. 14. Contamination at $100 \mu \mathrm{m}$ from faint stars. The solid histogram represents the derived contamination at $100 \mu \mathrm{m}$ from stars with fluxes below our flux cut. The dotted histogram shows the flux from stars explicitly removed from the maps.

The selective extinction is variable across optical passbands. This extinction curve is usually parameterized in terms of the $V$-band extinction $A(V)$ and a measure of the relative extinction between $B$ and $V$ band,

$$
R_{V}=\frac{A(V)}{A(V)-A(B)} \equiv \frac{A(V)}{E(B-V)} .
$$

The value of $R_{V}$ varies from 2.6 to 5.5 in the measurements of Clayton \& Cardelli (1988), with a mean value of 3.1 in the diffuse ISM. High-density regions are presumed to have larger values of $R_{V}$ as grains coagulate into larger grains that are relatively gray absorbers (Draine \& Anderson 1985; Guhathakurta \& Draine 1989; Kim \& Martin 1995). This variation is certainly a source of concern, as the extinction curves vary significantly in blue passbands for different values of $R_{V}$. These variations can be well represented by the same two-parameter function in any region of the Galaxy. We use the functional form of O'Donnell (1994) in the optical and that of Cardelli, Clayton, \& Mathis (1989) in the ultraviolet and infrared. The extinction curve is nearly the same at $\lambda>8000 \AA$ for all values of $R_{V}$, with deviations of up to $30 \%$ in the $V$ band and $55 \%$ in the $B$ band.

Fortunately, the observational evidence favors a single extinction curve for most diffuse clouds in the Galaxy, with $R_{V} \approx 3.1$. Even including stars in dense clouds, the 76 measurements of stars from Clayton \& Cardelli show a dispersion of 0.7 in $R_{V}$. This translates into a scatter of only $10 \%$ in $A(B) / A(\infty)$ and of $6 \%$ in $A(V) / A(\infty)$. Surprisingly, Kenyon, Dobrzycka \& Hartmann (1994) find agreement with an $R_{V}=3.1$ extinction curve for stars in the heavily reddened Taurus-Auriga cloud. Rieke \& Lebofsky (1984) have shown the extinction curve to be valid at wavelengths $1<\lambda<13 \mu \mathrm{m}$.

The extinction in each passband $b, \Delta m_{b}$, is evaluated for an extinction in the $V$ passband, $\delta m_{V}$ :

$$
\Delta m_{b}=-2.5 \log \left[\frac{\int d \lambda W_{b}(\lambda) S(\lambda) 10^{-\left(A_{\lambda} \Delta m_{V} / 2.5\right)}}{\int d \lambda W_{b}(\lambda) S(\lambda)}\right] .
$$

The system response, in terms of quantum efficiency, is represented by $W(\lambda)$, and $S(\lambda)$ is the photon luminosity of the source. As we have normalized the dust maps to the reddening of elliptical galaxies, we use an elliptical galaxy for the source. We average the normal elliptical galaxy SEDs from Kennicutt (1992), extrapolating the source as $S(\lambda) \propto \lambda$ outside his range of spectral coverage. The above expression has been evaluated for a variety of passbands in the limit of low extinction $\left(\Delta m_{V} \rightarrow 0\right)$, then rescaled to $A(V)=1$.

The system response is the convolution of the atmosphere, telescope optics, filter, and detector responses. Where possible, we have chosen the system responses that correspond to the commonly used standard stars for each filter. A photoelectric Cousins $U B V R_{\mathrm{KC}} I_{\mathrm{KC}}$ system is represented by the filter responses published by Landolt (1992) convolved with the quantum efficiency of the RCA 3103A photomultiplier and the atmospheric transparency at Cerro Tololo. A CCD Cousins $U B V R_{\mathrm{KC}} I_{\mathrm{KC}}$ system is represented by the filter responses of the CTIO TEK2 filter set, convolved with their Tek2K CCD Q.E. and the atmosphere at Cerro Tololo. The Stromgren $u b v \beta y$ system matches the filter curves published by Crawford \& Barnes (1970), convolved with a Tek2K CCD Q.E. and the atmosphere at Kitt Peak. The Gunn griz system matches the filter curves published by Schneider, Gunn, \& Hoessel (1983), convolved with the atmosphere at Palomar. The Spinrad night-sky filter $R_{\mathrm{S}}$ has been widely used at Lick Observatory and is convolved with the Orbit $2 \mathrm{~K}$ Q.E. and the atmosphere at Lick. The infrared $J H K L^{\prime}$ bands are represented by the IRCAM3 filter set at UKIRT, convolved with the atmosphere at Mauna Kea. The $u^{\prime} g^{\prime} r^{\prime} i^{\prime} z^{\prime}$ filter set for the Sloan Sky Survey represent the total system response at Apache Point as published by Fukugita et al. (1996). The total system response for some of the broadband filters for WFPC2 were taken from the Space Telescope Science Institute web page. The photographic responses for the Second Generation Digitized Sky Survey are from Weir, Djorgovski, $\&$ Fayyad (1995), convolved with the atmosphere at Palomar.

The $b_{J}$ passband used for the APM maps has an unusual definition and is treated differently. The APM maps were calibrated with CCD photometry using the following definition (Maddox et al. 1990a):

$$
b_{J} \equiv B-0.28(B-V) \text {. }
$$


Combining equations (B2) and (B3), one can show the following to be rigorously true:

$$
A\left(b_{J}\right)=0.72 A(B)+0.28 A(V) .
$$

The results for all passbands appear in Table 6 . The effective wavelength $\lambda_{\text {eff }}$ represents that wavelength on the extinction curve with the same extinction as the full passband. The final column normalizes the extinction to photoelectric measurements of $E(B-V)$. Assuming an $R_{V}=3.1$ dust model, the dust maps should be multiplied by the value in this column to determine the extinction in a given passband. Extinctions in narrow passbands can be determined by evaluating the Cardelli et al. or O'Donnell extinction law at the corresponding wavelength.

\section{APPENDIX C}

\section{DATA PRESENTATION}

The $100 \mu \mathrm{m}$ map and the dust map are available electronically. Both maps consist of the destriped, deglitched ISSA data recalibrated to DIRBE and point-source subtracted. The $100 \mu \mathrm{m}$ map maps the emission in units of $\mathrm{MJy} \mathrm{sr}^{-1}$. The dust map applies our temperature correction to convert $100 \mu \mathrm{m}$ emission to column density of dust, calibrated to $E(B-V)$ reddening in magnitudes. Reddenings and extinctions in other passbands are computed by multiplication with the numbers in Table 6 .

All maps are stored as FITS files in pairs of $4096 \times 4096$ pixel Lambert projections. The NGP projection covers the northern Galactic hemisphere, centered at $b=+90^{\circ}$, with latitude running clockwise. The SGP projection covers the southern Galactic hemisphere, centered at $b=-90^{\circ}$, with latitude running counterclockwise. (Note that Figs. 3, 8, 11, and 12 show the SGP projections rotated by $180^{\circ}$.) Galactic coordinates $(l, b)$ are converted to pixel positions $(x, y)$ via

$$
\begin{gathered}
x=2048 \sqrt{1-n \sin (b)} \cos (l)+2047.5, \\
y=-2048 n \sqrt{1-n \sin (b)} \sin (l)+2047.5,
\end{gathered}
$$

where $n=+1$ for the NGP, and $n=-1$ for the SGP. Pixel numbers are zero indexed, with the center of the lower left pixel having position $(x, y)=(0,0)$. These Lambert projections are minimally distorted at high Galactic latitudes, with the distortion approaching $40 \%$ at $b=0^{\circ}$. The pixel size of $\left(2{ }^{\prime} \cdot 372\right)^{2}$ well samples the FWHM of 6.1.

The caveats to using these maps to measure reddening or extinction can be summarized as follows:

1. Every effort has been made to remove both extragalactic sources and unresolved (Galactic) sources from the dust maps at $|b|>5^{\circ}$ and unconfused regions at lower latitudes. Some sources will remain, owing to confusion or unusual FIR colors. No sources fainter than $0.6 \mathrm{Jy}$ at $60 \mu \mathrm{m}$ are removed, although these are not significant contaminants.

2. The IRAS satellite did not scan a strip amounting to $3 \%$ of the sky. In addition, we remove a circle of radius $2^{\circ}$ centered at $(l, b)=(326.28,+51.66)$ that is contaminated by Saturn. These regions are replaced with DIRBE data and have no point sources removed.

\begin{tabular}{|c|c|c|c|c|c|c|c|}
\hline Filter & $\begin{array}{l}\lambda_{\text {eff }} \\
(\AA)\end{array}$ & $A / A(V)$ & $A / E(B-V)$ & Filter & $\begin{array}{c}\lambda_{\text {eff }} \\
\AA\end{array}$ & $A / A(V)$ & $A / E(B-V)$ \\
\hline Landolt $U \ldots \ldots$ & 3372 & 1.664 & 5.434 & Strömgren $u \ldots \ldots \ldots$ & 3502 & 1.602 & 5.231 \\
\hline Landolt $B \ldots \ldots$ & 4404 & 1.321 & 4.315 & Strömgren $b \ldots \ldots \ldots$ & 4676 & 1.240 & 4.049 \\
\hline Landolt $V \ldots \ldots$ & 5428 & 1.015 & 3.315 & Stromgren $v \ldots \ldots \ldots$ & 4127 & 1.394 & 4.552 \\
\hline Landolt $R \ldots \ldots$ & 6509 & 0.819 & 2.673 & Strömgren $\beta \ldots \ldots \ldots$ & 4861 & 1.182 & 3.858 \\
\hline Landolt $I \ldots \ldots$. & 8090 & 0.594 & 1.940 & Strömgren $y \ldots \ldots \ldots$ & 5479 & 1.004 & 3.277 \\
\hline CTIO $U \ldots \ldots .$. & 3683 & 1.521 & 4.968 & Sloan $u^{\prime}$ & 3546 & 1.579 & 5.155 \\
\hline CTIO $B \ldots \ldots \ldots$ & 4393 & 1.324 & 4.325 & Sloan $g^{\prime} \ldots$ & 4925 & 1.161 & 3.793 \\
\hline CTIO $V \ldots \ldots \ldots$ & 5519 & 0.992 & 3.240 & Sloan $r^{\prime}$ & 6335 & 0.843 & 2.751 \\
\hline CTIO $R$. & 6602 & 0.807 & 2.634 & Sloan $i^{\prime}$ & 7799 & 0.639 & 2.086 \\
\hline CTIO I . & 8046 & 0.601 & 1.962 & Sloan $z^{\prime}$ & 9294 & 0.453 & 1.479 \\
\hline UKIRT $J \ldots \ldots \ldots$ & 12660 & 0.276 & 0.902 & WFPC2 F300W. & 3047 & 1.791 & 5.849 \\
\hline UKIRT $H \ldots \ldots$. & 16732 & 0.176 & 0.576 & WFPC2 F450W ....... & 4711 & 1.229 & 4.015 \\
\hline UKIRT $K \ldots \ldots$ & 22152 & 0.112 & 0.367 & WFPC2 F555W...... & 5498 & 0.996 & 3.252 \\
\hline UKIRT $L^{\prime} \ldots \ldots$ & 38079 & 0.047 & 0.153 & WFPC2 F606W ....... & 6042 & 0.885 & 2.889 \\
\hline Gunn $g \ldots \ldots \ldots$ & 5244 & 1.065 & 3.476 & WFPC2 F702W ....... & 7068 & 0.746 & 2.435 \\
\hline Gunn $r \ldots \ldots \ldots$ & 6707 & 0.793 & 2.590 & WFPC2 F814W...... & 8066 & 0.597 & 1.948 \\
\hline Gunn $i \ldots \ldots \ldots$ & 7985 & 0.610 & 1.991 & DSS-II $g \ldots \ldots \ldots \ldots$ & 4814 & 1.197 & 3.907 \\
\hline Gunn $z \ldots \ldots \ldots$ & 9055 & 0.472 & 1.540 & DSS-II $r \ldots \ldots \ldots \ldots$ & 6571 & 0.811 & 2.649 \\
\hline Spinrad $R_{\mathrm{S}} \ldots \ldots$ & 6993 & 0.755 & 2.467 & DSS-II $i \ldots \ldots \ldots \ldots \ldots$ & 8183 & 0.580 & 1.893 \\
\hline $\mathrm{APM} b_{J} \ldots \ldots \ldots$ & 4690 & 1.236 & 4.035 & & & & \\
\hline
\end{tabular}

TABLE 6

ReLATIVE ExTinCtion For SeleCted BANDPASSES

NoтE.-Magnitudes of extinction evaluated in different passbands using the $R_{V}=3.1$ extinction laws of Cardelli et al. 1989 and O’Donnell 1994. The final column normalizes the extinction to photoelectric measurements of $E(B-V)$. 
3. The $100 \mu \mathrm{m}$ passband for the DIRBE satellite is somewhat different from the $100 \mu \mathrm{m}$ passband on IRAS. For a $20 \mathrm{~K}$ blackbody, the difference is small, but the color temperature of sources is sometimes very different than that of the cirrus. This sometimes results in artifacts in the temperature correction term (on scales of $1^{\circ}$ ). Our remedy is described in $\S 3.3$.

4. The LMC, SMC, and M31 are not removed from the maps, nor are sources within their Holmberg radius. Accurate reddenings through these galaxies are not possible, since their temperature structure is not sufficiently resolved by DIRBE. Typical reddenings toward these galaxies are estimated from the median dust emission in surrounding annuli: $E(B-V)=0.075 \mathrm{mag}$ for the LMC, $0.037 \mathrm{mag}$ for the SMC, and $0.062 \mathrm{mag}$ for M31.

5. At low Galactic latitudes $\left(|b|<5^{\circ}\right)$, most contaminating sources have not been removed from the maps, and the temperature structure of the Galaxy is not well resolved. Furthermore, no comparisons between our predicted reddenings and observed reddening have been made in these regions. Thus, our predicted reddenings here should not be trusted, though inspection of the maps might be of some use.

6. The normalization of the dust column density to reddening has a formal uncertainty of $10 \%$.

7. Should one wish to change the DIRBE/IRAS dust zero point to be consistent with the Burstein-Heiles maps, we suggest subtracting $0.020 \mathrm{mag}$ in $E(B-V)$.

The reddening maps will be available in the CD-ROM series of the AAS or from the web site. ${ }^{2}$ Mask files are also available that contain the most important processing steps for any given position on the sky. Further details will be available with the data files.

\footnotetext{
${ }^{2}$ http://astro.berkeley.edu/davis/dust/index.html.
}

Arendt, R. G., et al. 1998, in preparation

Boggess, N. W., et al. 1992, ApJ, 397, 420

Bond, J. R., Carr, B. J., \& Hogan, C. J. 1991, ApJ, 367, 420 1986, ApJ, 306, 428

Boulanger, F., Abergel, A., Bernard, J. P., Burton, W. B., Desert, F. X., Hartmann, D., Lagache, G., \& Puget, J. L. 1996, A\&A, 312, 256

Burstein, D., Davies, R. L., Dressler, A., Faber, S. M., Stone, R. P. S.,

Lynden-Bell, D., Terlevich, R. J., \& Wegner, G. 1987, ApJ, 64, 601

Burstein, D., \& Heiles, C. 1978, ApJ, 225, 40 (BH)

1982, AJ, 87, 1165

Cardelli, J. A., Clayton, G. C., \& Mathis, J. S. 1989, ApJ, 345, 245

Cao, Y., Prince, T. A., Tereby, S., \& Beichman, C. A. 1996, PASP, 108, 535

Chan, F. K., \& O'Neill, E. M. 1975, Feasibility Study of a Quadrilaterized Spherical Cube Earth Data Base (Silver Spring, MD: Computer Sciences Corp.)

Clayton, G. C., \& Cardelli, J. A. 1988, AJ, 96, 695

COBE Diffuse Infrared Background Experiment (DIRBE) Explanatory Supplement. 1995, ed. M. G. Hauser et al. (Greenbelt, MD: NASA/ GSFC), available from the NSSDC

Crawford, D. L., \& Barnes, J. V. 1970, ApJ, 75, 978

de Oliveira-Costa, A., Kogut, A., Devlin, M., Netterfield, C. B., Page, L. A., \& Wollack E. J. 1997, ApJ, 482, L17

de Vaucouleurs, G., et al. 1991, Third Reference Catalogue of Bright Galaxies (New York: Springer) (RC3)

Draine, B. T., \& Anderson, N. 1985, ApJ, 292, 494

Draine, B. T., \& Lazarian, A. 1997, preprint, astro-ph/9710152

Draine, B. T., \& Lee, H. M. 1984, ApJ, 285, 89

Faber, S. M., Wegner, G., Burstein, D., Davies, R. L., Dressler, A., LyndenBell, D., \& Terlevich, R. J. 1989, ApJS, 69, 763

Finkbeiner, D. P., Schlegel, D. J., \& Davis, M. 1997, in preparation

Fisher, K. B., Huchra, J. P., Strauss, M. A., Davis, M., Yahil, A., \& Schlegel, D. $1995, \mathrm{ApJS}, 100,69$

Fixsen, D. J., et al. 1997, ApJ, 490, 482

Fixsen, D. J., Dwek, E., Mather, J. C., Bennett, C. L., \& Shafer, R. A. 1998, ApJ, in press

Franceschini, A., et al. 1997, preprint, astro-ph/9707080

Fukugita, M., Ichikawa, T., Gunn, J. E., Doi, M., Shimasaku, K., \& Schneider, D. P. 1996, AJ, 111, 1748

Gaustad, J. E., McCullough, P. R., \& Van Buren, D. 1996, PASP, 108, 351

Guhathakurta, P., \& Draine, B. T. 1989, ApJ, 345, 230

Guiderdoni, B., Bouchet, F. R., Puget, J. L., Lagache, G., \& Hivon, E. 1997, preprint, astro-ph/9706095

Hartmann, D., \& Burton, W. B. 1997, Atlas of Galactic Neutral Hydrogen (Cambridge: Cambridge Univ. Press)

Hartmann, D., Magnani, L., \& Thaddeus, P. 1997, preprint

Hauser, M. 1996, in AIP Conf. Proc. 348, Unveiling the Cosmic IR Background, ed. E. Dwek (New York: AIP), 11

Hauser, M., et al. 1998, in preparation

Heiles, C. 1976, ApJ, 204, 379

Herbstmeier, U., Heithausen, A., \& Mebold, U. 1993, A\&A, 272, 514

Hilditch, R. W., Hill, G., \& Barnes, J. V. 1983, MNRAS, 204, 241

IRAS Catalogs and Atlases: Explanatory Supplement. 1988, ed. Beichman et al. (Washington, DC: US Government Printing Office)

Jaffe, A. H., Finkbeiner, D. P., \& Bond, J. R. 1998, in preparation

Joint IRAS Science Working Group. 1988, IRAS Point Source Catalog,

Version 2 (Washington, DC: US Government Printing Office)

Kelsall, T., et al. 1998, in preparation

\section{EFERENCES}

Kennicutt, R. C. 1992, ApJS, 79, 255

Kenyon, S. J., Dobrzycka, D., \& Hartmann, L. 1994, AJ, 108, 1872

Kim, H., \& Martin, P. 1995, ApJ, 462, 296

Kogut, A., Banday, A. J., Bennett, C. L., Gorski, K. M., Hinshaw, G., \& Reach, W. T. 1996, ApJ, 460, 1

Landolt, A. U. 1992, AJ, 104, 340

Leitch, E. M., Readhead, A., Pearson, T., \& Myers, S. 1997, preprint, astro-ph/9705241

Lockman, F. J., Jahoda, K., \& McCammon, D. 1986, ApJ, 302, 432

Low, F. J., et al. 1984, ApJ, 278, L19

Madau, P. 1997, preprint, astro-ph/9709147

Maddox, S. J., Efstathiou, G., \& Sutherland, W. J. 1990a, MNRAS, 246, 433

Maddox, S. J., Sutherland, W. J., Efstathiou, G., \& Loveday, J. 1990b, MNRAS, 243, 692

Magnani, L., Blitz, L., \& Mundy, L. 1985, ApJ,295,402

Malkan, M. A. \& Stecker, F. W. 1997, preprint, astro-ph/9710072

Mathis, J. S., \& Cardelli, J. A. 1992, ApJ, 398, 610

Mebold, U., Cernicharo, J., Velden, L., Reif, K., Crezelius, C., \& Goerigk, W. 1985, A\&A, 151, 427

Neugebauer, G., et al. 1984, ApJ, 278, L1

O'Donnell, J. E. 1994, ApJ, 422, 1580

Peebles, P. J. E. 1971, Physical Cosmology (Princeton, NJ: Princeton Univ. Press), 59

Perry, C. L., \& Christodoulou, D. M. 1996, PASP, 108, 772

Perry, C. L., \& Johnston, L. 1982, ApJS, 50, 451

Pettini, M., Steidel, C. C., Adelberger, K. L., Kellogg, M., Dickinson, M., \& Giavalisco, M. 1997, preprint, astro-ph/9708117

Postman, M., \& Lauer, T. R. 1995, ApJ, 440, 28

Puget, J. L., et al. 1996, A\&A, 308, L5

Reach, W. T., et al. 1995, ApJ, 451, 188 1996, A\&A, 315, 381

Reynolds, R. J. 1990, in IAU Symp. 144, The Interstellar Disk-Halo Connection in Galaxies, ed. H. Bloeman (Dordrecht: Kluwer), 67

Reynolds, R. J., Tufte, S. L., Kung, D. T., McCullough, P. R., \& Heiles, C. 1995, ApJ, 448, 715

Rice, W., et al. 1988, ApJS, 68, 91

Rieke, G. H., \& Lebofsky, M. J. 1984, ApJ, 288, 618

Rowan-Robinson, M., Jones, M., Leech, K., Vedi, K., \& Hughes, J. 1991, MNRAS, 249, 729

Schlegel, D. J. 1995, Ph.D. thesis, University of California at Berkeley

Schneider, D. P., Gunn, J. E., \& Hoessel, J. G. 1983, ApJ, 264, 337

Simonetti, J. H., Dennison, B., \& Topsana, G. A. 1996, ApJ, 458, L1

Sodroski, T. J., Odegard, N., Arendt, R. G., Dwek, E., Weiland, J. L., Hauser, M. G., \& Kelsall, T. 1997, ApJ, 480, 173

Spaans, M., \& Neufeld, D. A. 1997, ApJ, 484, 785

Stark, A. A., Gammie, C. F., Wilson, R. W., Bally, J., Linke, R. A., Heiles, C., \& Hurwitz, M. 1992, ApJS, 79, 77

Strauss, M. A., Davis, M., \& Huchra, J. P. 1990, ApJ, 361, 49

Strauss, M. A., Huchra, J. P., Davis, M., Yahil, A., Fisher, K., \& Tonry, J. 1992, ApJS, 83, 29

Teerikorpi, P. 1990, A\&A, 235, 362

Vogeley, M. 1997, preprint, astro-ph/9711209

Weir, N., Djorgovski, S., \& Fayyad, U. M. 1995, AJ, 110, 1

Wheelock, S. L., et al. 1994, IRAS Sky Survey Atlas: Explanatory Supplement (Pasadena: JPL 94-11)

Zepf, S. E., \& Silk, J. 1996, ApJ, 466, 114 
Note added in proof.-The Burstein \& Heiles reddening map appears to have high accuracy in the northern sky $\left[\delta(1950)>-23^{\circ}\right]$, where galaxy counts were used to model gas/dust variations. Splitting the data into two subsamples, one finds $f=0.25$ for the 257 galaxies at $\delta(1950)>-23^{\circ}$ and $f=0.31$ for the 115 galaxies at $\delta(1950)<-23^{\circ}$. Burstein et al. (1987) identify one region in the southern sky, approximately bounded by $l=\left[230^{\circ}, 310^{\circ}\right], b=\left[-20^{\circ}, 20^{\circ}\right]$, where a low dust-to-gas ratio is presumed to make the $\mathrm{BH}$ predictions too large. We find that the dust-to-gas ratio in this region is variable, but not unusual on average. Comparing the BH map to Leiden-Dwingeloo $21 \mathrm{~cm}$ data in the South, we conclude that the $\mathrm{BH}$ map may be unreliable near their survey edges at $b= \pm 10^{\circ}$. Within the region identified by Burstein et al., we find a smaller region, bounded by $l=\left[230^{\circ}, 240^{\circ}\right], b=\left[-15^{\circ},-10^{\circ}\right]$, which appears corrupted and too high in the BH map. 